First publ. in: Journal of Rheology 49 (2005), 1, pp. 237-269

\title{
Glass transitions and shear thickening suspension rheology
}

\author{
C. B. Holmes and M. E. Cates ${ }^{\text {a) }}$ \\ School of Physics, The University of Edinburgh, JCMB, The King's Buildings, \\ Edinburgh, EH9 3JZ United Kingdom
}

M. Fuchs

Fachbereich Physik, Universität Konstanz, D-78457 Konstanz, Germany

P. Sollich

Department of Mathematics, King's College, University of London, Strand, London, WC2R 2LS United Kingdom

(Received 29 June 2004; final revision received 10 September 2004)

\section{Synopsis}

We introduce a class of simple models for shear thickening and/or "jamming" in colloidal suspensions. These are based on the schematic mode coupling theory (MCT) of the glass transition, having a memory term that depends on a density variable, and on both the shear stress and the shear rate. (Tensorial aspects of the rheology, such as normal stresses, are ignored for simplicity.) We calculate steady-state flow curves and correlation functions. Depending on model parameters, we find a range of rheological behaviors, including "S-shaped" flow curves, indicating discontinuous shear thickening, and stress-induced transitions from a fluid to a nonergodic (jammed) state, showing zero flow rate in an interval of applied stress. The shear thickening and jamming scenarios that we explore appear broadly consistent with experiments on dense colloids close to the glass transition, despite the fact that we ignore hydrodynamic interactions. In particular, the jamming transition we propose is conceptually quite different from various hydrodynamic mechanisms of shear thickening in the literature, although the latter might remain pertinent at lower colloid densities. Our jammed state is a stress-induced glass, but its nonergodicity transitions have an analytical structure distinct from that of the conventional MCT glass transition.

\section{INTRODUCTION}

Under flow, concentrated hard sphere suspensions can shear thicken: the measured viscosity increases with the applied stress or flow rate [Bender and Wagner (1996), d'Haene et al. (1993), Frith et al. (1996), Laun (1994), O'Brien and Mackay (2000)]. The effect can be pronounced, in some cases giving an order-of-magnitude jump in the shear stress on exceeding a "critical" shear rate. In such "discontinuous" shear thickening the flow often becomes erratic, suggesting the continual formation and breakup of stresssupporting structures [Frith et al. (1996)]. This idea is supported by recent pressure-

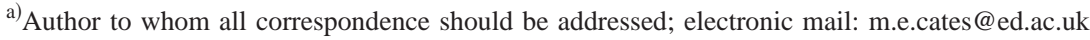


driven flow experiments on model colloids, in which transient solid regions are directly visualized [Haw (2004)]. Computer simulations give a similar picture, with simulations at infinite Peclet number suggesting that, in the absence of Brownian motion, a static, load bearing jam may form [Melrose and Ball (1995)]. This is reminiscient of jamming in granular materials, in which the solidity of the material may result from the applied stress itself [Cates et al. (1998)].

The notion that strongly shear thickened states have the character of an amorphous solid, or glass, is supported by recent experiments [Bertrand et al. (2002)], in which a droplet of shear thickening material undergoes a fluid-solid transition upon shearing. In these experiments, a suspension (mean particle radius $a \approx 3 \mu \mathrm{m}$ ) was transformed into a solid state, simply by stirring with a spatula. The jammed solid persists after cessation of stirring, but is refluidized by vibration. Also, it is reported that introducing a droplet of the quiescent liquid state adjacent to a jammed droplet (at the same concentration) results in the two coalescing to form a larger fluid droplet. The description of these phenomena reported in Bertrand et al. (2002) is brief; moreover the system of Bertrand et al. (2002) is not hard-sphere like, probably non-Brownian and the reported phenomena are at relatively modest concentrations. [Very similar observations have been made in suspensions of zeolite particles (Warren).] However, similar phenomena have also been observed in a model system that closely approaches the idealization of Brownian hard-sphere colloids [Haw (2004)]. For hard spheres, the relevant concentration range lies above 50\% by volume.

Thus there is some evidence of two distinct states of a dense suspension, one fluid and one jammed. These must presumably have different internal organization. Indeed, the surface of jammed droplets appears dry or dull and "pasty" (becoming shiny again only when refluidized by vibration or coalescence) suggesting that partial emergence of particles is needed to sustain the jammed state [Bertrand et al. (2002), Haw (2004)]. This is consistent with the presence of large, static deviatoric stresses within the bulk of a jammed droplet, balanced at the surface by capillary forces.

Such experiments pose important challenges to the rheological modeling of colloidal suspensions. In particular they suggest, though they do not prove, that static shear stresses might be sustained indefinitely in a bulk sample at rest in a rheometer-even if the same sample is fluid at lower stresses. (In this paper we address only this bulk geometry.) On the other hand, shear thickening is commonly attributed to "hydrodynamic clustering" [Ball and Melrose (1995), Bergenholtz et al. (2002), Brady (1996), Melrose and Ball (1995)] - that is, the formation of transient clusters of particles in lubrication contact. This results in large hydrodynamic stresses in fast flowing suspensions, and there is simulation and other evidence that these are the dominant stresses in the shear-thickened regime [Bender and Wagner (1996), Brady (1996), Phung et al. (1996)]. However, some of these simulations are limited to relatively modest volume fractions of colloid $(\phi \leqslant 0.49)$. A wider range of volume fractions has been studied experimentally in a model hard-sphere colloid [O'Brien and Mackay (2000)]. These authors use stress-jump studies to argue that at low volume fractions shear-thickening originates in hydrodynamic stresses but that at high ones $(\phi \geqslant 0.59)$ an "elastic-like" mechanism takes over. Thus there is clear evidence, even in model systems, for physics beyond that of hydrodynamic clustering in shear-thickening at high volume fraction. \{Layer formation has been proposed as such an additional mechanism [Hoffman (1998)].\} In any case it seems highly unlikely that the "static jamming" observed by Bertrand et al. (2002) is due to hydrodynamic interactions alone, since in the persisting solid phase there is no macroscopic flow to provide those interactions. Also, as far as we know, hydrodynamic models for shear thickening offer no immediate explanation of nonmonotonic regions of the flow curve. 
Such regions may be observed directly [Bertrand et al. (2002), Frith et al. (1996)], and can lead \{via well understood shear-banding mechanisms [Olmsted (1999)]\} to discontinuous shear thickening and accompanying hysteresis [Laun (1994)].

Although discontinuous shear thickening is not limited to regimes where nonhydrodynamic stresses dominate, it nonetheless seems clear that mechanisms other than pure hydrodynamics are at work in sufficiently dense shear-thickening suspensions [O'Brien and Mackay (2000)]. Indeed, it has already been emphasised [Ball and Melrose (1995), Melrose and Ball (1995)] that deviations from pure lubrication forces can dominate the physics of any hydrodynamically clustered state. \{These deviations could arise, for example, from deformation of stabilizing polymer layers around the particles [Ball and Melrose (1995), Melrose (2003), Melrose and Ball (1995), Melrose et al. (1996)].\} Crucially, hydrodynamic theories neglect something else too: the colloidal glass transition [Pusey (1989)].

In quiescent colloidal fluids, Brownian relaxation processes are often interpreted in terms of particle "caging." There is a short-time relaxation, called $\beta$-relaxation, which is a many-body process during which particles remain within a cage of nearest neighbors. This is followed by a late-time $\alpha$-relaxation, which corresponds to particles escaping their cages. As the glass transition is approached, the cages become tighter, and both of these relaxation times become longer. In quiescent dense suspensions, it is this mechanism (rather than simply the vanishing of mobility as interparticle gaps close to zero separation at random close packing) that leads to the observed slow relaxations and large viscosities. Within the so-called mode coupling theory (MCT) of the glass transition, the divergence of the $\alpha$-relaxation time (and, within MCT, also the viscosity) occurs at a colloid volume fraction $\phi_{g}$ around 52\% (although this value depends on the input static structure factor). This is far below the random close packing density $(64 \%)$ which is the natural location of any divergence in purely hydrodynamic theories. In practice, this MCT value is too low, but not by much-light scattering data on $\alpha$-relaxation locate the glass transition at $58 \%$ [van Megen and Underwood (1994)].

We contend that the slow relaxation of thermodynamic forces caused by particle caging remains important in sheared dense suspensions, and is key to understanding their non-Newtonian rheology. If this is true, the interesting physics should arise at shear rates $\dot{\gamma}$ set by a renormalized Peclet number $\dot{\gamma} \tau_{\alpha}$ involving the $\alpha$-relaxation time. In contrast, hydrodynamic mechanisms are controlled, in the simplest approximation, by the bare Peclet number $\dot{\gamma} \tau_{0}$, with $\tau_{0}$ the diffusive relaxation time of an isolated particle [Bender and Wagner (1996), Phung et al. (1996)]. Less naively, one could expect this to be renormalized to $\dot{\gamma} \tau_{1}$ where $\tau_{1}$ is a lubrication-diffusion time that diverges at random close packing [Foss and Brady (2000), O'Brien and Mackay (2000)]. But as emphasized before, even $\tau_{1}$ is vastly shorter than $\tau_{\alpha}$ in fluids close to the glass transition, and infinitely shorter throughout the glass phase itself, where $\tau_{\alpha}$ has diverged.

While in practice the hydrodynamic and thermodynamic forces in very dense colloids may be interdependent, this separation of time scales makes it legitimate to explore the possible connection between the glass transition and shear thickening in its own right, ignoring hydrodynamic interactions in the first instance. From this viewpoint, the jammed state found experimentally is a candidate for a stress-induced, anisotropic colloidal glass. Such a state could arise if the applied stress alters the material's structure, leading to an increased number of close contacts, hindering diffusion and promoting dynamical arrest through the tightening of the cage around each particle.

In this picture, as in a conventional glass, solidity is due to thermodynamic forces (purely entropic for hard spheres): deformation of the material results in a free energy penalty which appears macroscopically as elasticity. An alternative is that Brownian 
motion is sufficiently weak that these entropic forces are irrelevant, leaving interparticle forces alone to resist deformation: in hard spheres, stress applied to the jammed solid is then transmitted by direct contacts. In this case, the jamming more closely resembles that of a granular medium [Cates et al. (1998)]. [In fact it has been suggested by Liu and Nagel (1998) that, even in this non-Brownian case, arrest is related to a glass transition; we do not pursue this here.] In a jammed suspension, it is not clear which mechanism dominates; the answer might depend strongly on particle size. Nor is it clear under what conditions static jamming may be achieved: sufficient Brownian motion might give all or most jammed states a finite lifetime. Note that, in contrast to some authors [for example Liu and Nagel (1998)], in this work we use the word jamming to mean an arrest transition caused specifically by imposed stress rather than other causes, such as attractive interactions.

Our modeling strategy, which was outlined with selected results in Holmes et al. (2003), neglects hydrodynamics, and assumes a dynamical glass transition picture rather than one based on direct mechanical contact between particles [Cates et al. (1998)]. It builds upon a schematic version of MCT, and is guided by more formal (and less schematic) MCT work on the role of shear in the glass transition [Fuchs and Cates (2002, 2003)]. The latter formal approach currently gives only shear-thinning behavior, or straininduced fluidisation. To this we add a new feature in our schematic models: stressinduced arrest. (Capturing the same physics, beyond the schematic level, remains an open issue for future development of the more formal theory [Cates (2003)].) In combination, these two features suggest various shear thickening and jamming scenarios, some of which appear broadly consistent with the experimental features noted earlier.

In some but not all cases, these scenarios are similar to ones found before from a quite different approach to glassy rheology, originally intended for "soft glasses" (foams, dense emulsions) rather than colloidal suspensions. In particular, Head et al. $(2001,2002)$ incorporated an ad hoc local jamming effect into the "soft glassy rheology model" (SGR) [Cates and Sollich (2004), Fielding et al. (2000), Sollich et al. (1997)] thereby converting its shear-thinning behavior into a shear thickening one. Our work on schematic MCT, though equally ad hoc at this stage, has the advantage that one day it might be underpinned by full MCT-type calculations, whose form might well be guided by comparing our different schematic model variants with experimental data. (A drawback is that, unlike SGR-based work, we can only address steady state properties and not dynamical ones.) Some of the flow curves we obtain are also related to those found by Hess and Hess (1994) although their work did not attempt to relate shear thickening to glass transitions.

The rest of this paper is organized as follows. Section II provides a brief introduction to MCT; in Sec. III, we formulate our new class of schematic models. Analytical and numerical methods are described in Sec. IV and the results of these presented in Secs. V and VI. Section VII contains a discussion of our results in the context of experiment, and we conclude in Sec. VIII.

\section{MODE COUPLING THEORY}

For a comprehensive description of MCT, see Götze (1989). Here, we outline only those aspects pertinent to the current work. The central quantities of the theory are the Fourier-space density fluctuations at wave vector $\mathbf{q}, \delta \rho(\mathbf{q}, t)$. The correlators of these quantities, $\phi_{\mathbf{q}}(t) \equiv\langle\delta \rho(\mathbf{q}, t) \delta \rho(-\mathbf{q}, 0)\rangle /\left\langle|\delta \rho(\mathbf{q})|^{2}\right\rangle$ may be measured in scattering experiments [Pusey (1989)], and describe the system's dynamics. In a liquid, the system is ergodic and $\phi_{\mathbf{q}}(t)$ decays to zero with time for all $\mathbf{q}$. In a glass it does not: 
$\lim _{t \rightarrow \infty} \phi_{\mathbf{q}}(t)=f_{\mathbf{q}}>0$, where the nonergodicity parameters $f_{\mathbf{q}}$ characterize the arrest. A finite $f_{\mathbf{q}}$ implies inability to relax on a lengthscale $\sim 2 \pi / q$. (In general, all $f_{\mathbf{q}}$ become nonzero at the same transition point; once one Fourier component of density is frozen, the others see this as a random potential and acquire nondecaying mean values.)

Within MCT, equations of motion are found for the correlators $\phi_{\mathbf{q}}(t)$. Assuming overdamped local motion appropriate to colloids, and dropping $\mathbf{q}$ subscripts, the result is [Götze (1998)]:

$$
\phi(t)+\tau_{0} \dot{\phi}(t)+\int_{0}^{t} m\left(t-t^{\prime}\right) \dot{\phi}\left(t^{\prime}\right) \mathrm{d} t^{\prime}=0,
$$

where $\tau_{0}$ is a time scale set by the microscopic dynamics. Here $m\left(t-t^{\prime}\right)$ is the memory function, and describes a retarded friction which, in the colloidal glass transition, arises by caging of a particle by its neighbors. In MCT, the memory function is found approximately by integrating (over wave vectors) a quadratic product of correlators, with coupling constants that depend solely on the static structure factor $S(q)=(1 / N)$ $\left\langle|\delta \rho(\mathbf{q})|^{2}\right\rangle$ of the system. The $\tau_{0}$ term represents instantaneous solvent friction (with many-body hydrodynamics omitted) and gives exponential relaxation of correlations in dilute colloids, in which memory effects are negligible. Note that the nature of the interparticle interactions enters through $S(q)$; MCT is not limited to hard-sphere colloids.

In MCT, the zero-shear viscosity diverges with the $\alpha$-relaxation time of the density correlations. An infinitesimal stress applied to a colloidal glass state gives zero steadystate shear rate $\dot{\gamma}$ : glasses are solid, although creep (sublinear in time) is not excluded. The extension of MCT to finite $\dot{\gamma}$ requires care, as Eq. (1) relies upon the fluctuation dissipation theorem (FDT) [Bouchaud et al. (1996)] which need not hold under shear [Barrat and Berthier (2000)]. However, extended forms of MCT describing shear have been formulated, either with the assumption of a FDT-like condition [Miyazaki and Reichmann (2002)], or without it [Fuchs and Cates $(2002,2003)$ ]. These approaches lead to equations of motion for correlators like $\phi_{\mathbf{q}}$ (redefined to account for simple advection) which formally resemble those of the unsheared theory. Solution of these equations, performed via MCT-like approximations, leads to the conclusion that structural relaxation always speeds up in a flowing system: $\tau_{\alpha}(\dot{\gamma}) \leqslant \tau_{\alpha}(0)$. \{Analogous behavior is seen in mean field spin models of driven glasses [Berthier et al. (2000)].\} Physically, this is because small scale diffusive motions are magnified by advection, facilitating break up of the cage on a time scale set by $1 / \dot{\gamma}$. In $\mathbf{q}$ space, the important fluctuations, which have wave vectors near the peak of $S(q)$, are advected on this time scale to higher $q$ where they decay rapidly. Even those fluctuations which are not directly advected become ergodic [Fuchs and Cates $(2002,2003)]$.

So far then, these microscopic extensions of MCT to sheared systems do not predict jamming under shear stress. This may be because shear thickening requires hard-core particle interactions which are not fully captured by the harmonic approximation to the thermodynamic forces conventionally made in MCT [Cates (2003)]. The latter is tantamount to an expansion of the free energy to second order in density fluctuations; in order to see the jamming effect, one might have to go beyond this order. We do not attempt this formidable task here: rather, we adopt a simpler approach, beginning from "schematic models" of the MCT glass transition. These stripped-down models represent a gross simplification of the full theory and yet manage to capture many of the key features of the quiescent glass transition [Götze (1989)]. In them, one considers a single correlator $\phi(t)$ (which may be considered to represent fluctuations at some typical wave vector), rather than the infinite set $\phi_{\mathbf{q}}(t)$. The memory function is then written as a polynomial of this 
correlator, with coefficients (coupling constants) that encode the interactions between particles (just as $S(q)$ encodes these in full MCT). Larger coupling constants correspond to higher colloid densities and/or lower temperatures.

Of interest here are two schematic models, known as the F2 and F12 models, defined by a shared equation [Eq. (1)] for the correlation function $\phi(t)$, and by the memory functions

$$
\begin{gathered}
m(t)=v_{2} \phi^{2}(t)(\mathrm{F} 2 \text { model }), \\
m(t)=v_{1} \phi(t)+v_{2} \phi^{2}(t)(\mathrm{F} 12 \text { model }) .
\end{gathered}
$$

The F2 model is chosen mainly for calculational simplicity whereas F12 captures more of the analytic structure of the full MCT picture. Each model exhibits a glass transition with increasing $v_{n}$; for the F2 model, this occurs at $v_{2}^{c}=4$ while in the F12 model \{which has $v_{2}>1$ [Götze (1989)]\}, there is a locus of transition points given by $v_{1}^{c}$ $=v_{2}\left(\sqrt{4 / v_{2}}-1\right)$. As these transitions are crossed, the single nonergodicity parameter $f \equiv \phi(\infty)$ jumps discontinuously, from zero on the liquid side of the transition, to a finite value $f_{c}$ on the glass side. On further increasing the coupling, there is a nonanalytic (square root) increase in $f$ beyond $f_{c}$. This behavior of $f$ is faithful to the full MCT in which a similar discontinuity and square root law are found for the $f_{\mathbf{q}}$.

\section{RHEOLOGICAL MODELS}

To incorporate shearing into the schematic MCT models we need to address two distinct effects. First, flow erodes memory: particles with nonzero separation in the flow gradient direction become separated in the flow direction on times $t \sim 1 / \dot{\gamma}$. Thus, diffusive motion is accentuated by the flow, with the result that cages are broken and correlations decay to zero. The second effect is that stress (as distinct from flow) can alter the local caging of particles such as to promote arrest. Within a schematic theory, the latter requires us to postulate a stress dependence of the coupling constants. Perhaps the simplest idea is to assume a Taylor expansion of the coupling constants $v_{n}(n=1,2)$ in Eqs. (2) and (3), that is, $v_{n}(\sigma)=v_{n}+\Sigma_{k} \alpha_{k} \sigma^{k}$. One could then neglect odd powers on the grounds of symmetry. However, this approach is limited to the region around $\sigma=0$, whereas we are interested in nonzero stresses (in particular, values close to the yield stress of an incipient glass). Also, it turns out that the topology of flow curves depends strongly on the form of $v_{n}(\sigma)$ at large $\sigma$, rather than that near the origin. Hence, although we choose mainly simple power laws for $v_{n}(\sigma)$, these are not to be viewed as a Taylor expansion around $\sigma=0$. In particular, a linear dependence $v_{n}(\sigma)=v_{n}+\alpha \sigma$, is not excluded by symmetry. Here, and from now on, $\sigma$ denotes the absolute value of the shear stress. The various model forms chosen for $v_{n}(\sigma)$ are specified below in Eqs. (7)-(11).

To allow for the flow-induced memory loss, the memory function should also be attenuated by a strain-dependent factor [Fuchs and Cates $(2002,2003)]$. This suggests that we write

$$
m(t)=\sum_{n} v_{n}(\sigma) \phi^{n}(t) f(\dot{\gamma} t)
$$

where the function $f(\dot{\gamma} t)$ is monotonically decreasing. Microscopic theory [Fuchs and Cates $(2002,2003)]$ suggests a specific form: $f(\dot{\gamma} t)=\left(1+\dot{\gamma}^{2} t^{2}\right)^{-1}$. But for algebraic convenience, we shall mainly consider an exponential form $f(\dot{\gamma} t)=\exp (-\dot{\gamma} t)$. 
In Eq. (4) for the memory function, the effect of shearing appears in two quite separate ways: via the applied stress $\sigma$, and with the resulting (steady state) shear rate $\dot{\gamma}$. These two quantities are related through the viscosity

$$
\eta \equiv \sigma / \dot{\gamma}
$$

In order to close the model, we now need a prescription for the viscosity. In linear response theory, the shear viscosity is expressed as the time integral of a stress correlator [Hansen and McDonald (1976)]. In our simplified model, we have but one correlator, and so we choose schematically to identify the viscosity as

$$
\eta=\int_{0}^{\infty} \phi(t) \mathrm{d} t=\tau
$$

where the appropriate elastic modulus has been set equal to unity, rendering the stress dimensionless. Equation (6) is somewhat ad hoc, since we are not studying the linear response regime, but experiment suggests a close relationship between viscosity and relaxation times [Meeker et al. (1997)]. Equation (6) means that dynamical arrest (nonzero $f$ ) implies a divergent viscosity, and hence zero shear rate, in steady state. This is fully consistent with arguments made earlier that finite $\dot{\gamma}$ precludes arrest. Note that equating $\eta$ to an integral of $\phi^{2}(t)$ (as is more in line with the full version of MCT) would give very similar results.

Equations (1), (4), and (6) are shared by all the models in the class we study here. Different members of the class have different choices of $v_{n}(\sigma)$ and $f(\dot{\gamma} t)$ in Eq. (4). Among the variants we shall address are

$$
\begin{gathered}
m(t)=\left(v_{2}+\alpha \sigma\right) \phi^{2}(t) \exp (-\dot{\gamma} t) \text { model I, } \\
m(t)=\left(v_{2}+\alpha \sigma^{2}\right) \phi^{2}(t) \exp (-\dot{\gamma} t) \text { model II. }
\end{gathered}
$$

These variants are amenable to an analytic approach, which we present in the next section. We also consider a model III which interpolates between model II at small stresses and model I at large ones; this has

$$
m(t)=\left(v_{2}+\alpha \inf \left[\sigma, \sigma^{2}\right]\right) \phi^{2}(t) \exp (-\dot{\gamma} t) \text { model III. }
$$

The other variations which we shall study are

$$
\begin{gathered}
m(t)=\left(v_{2}+\alpha \sigma\right) \phi^{2}(t) /\left(1+\dot{\gamma}^{2} t^{2}\right) \text { model IV } \\
m(t)=\left[\left(v_{1}+\alpha_{1} \sigma\right) \phi(t)+\left(v_{2}+\alpha_{2} \sigma\right) \phi^{2}(t)\right] \exp (-\dot{\gamma} t) \text { model V. }
\end{gathered}
$$

Model IV differs from model I only in that the choice of $f(\dot{\gamma} t)$ is closer to the form expected from the full MCT [Fuchs and Cates $(2002,2003)]$. Unfortunately this limits analytic progress, but we present numerical results for this case later. Model V departs from variants I-IV by being based on the F12 model rather than F2; similar remarks apply.

In each of these model variants, there are control parameters such as $v_{n}$ and $\alpha$ : these are expected to have nontrivial dependence on both the concentration of colloids and their interactions. Since in practice a range of different shear-thickening scenarios are observed for different materials, an attempt at a parameter-free theory would, at this stage, be misguided. We choose $\alpha \geqslant 0$ in this paper. 


\section{SOLVING THE MODELS}

\section{A. Analytical developments}

Our analytical work is based on the F2 model and, hence, restricted to models I-IV, for which $v_{1}=0$. The methods are adapted from standard techniques used in solution of the MCT equations of motion \{see, e.g., [Götze (1989)]\}. We begin by defining a Laplace transform

$$
f(z) \equiv i \int_{0}^{\infty} f(t) \exp (i z t) \mathrm{d} t ; \quad \Im(z)>0 .
$$

Then, Laplace transforming Eq. (1), we obtain

$$
\phi(z)=\frac{-1}{z-\frac{1}{i \tau_{0}+m(z)} .}
$$

The dynamical slowing down associated with the glass transition is contained in $m(z)$. In contrast, the instantaneous friction term remains constant, and so, close to the transition, $\left|\tau_{0}\right| \ll|m(z)|$ for low frequencies. For clarity in the following, we label the position in parameter space by the vector $\mathbf{v} \equiv\left(v_{2}, \alpha, \sigma\right)$ so that, e.g., $\phi_{\mathbf{v}}(z)$ is the frequency-space correlator at frequency $z$ for parameters given by $\mathbf{v}$. (Likewise, $\dot{\gamma}_{\mathbf{v}}$ is the shear rate for the given parameter values.) At low frequencies then, we neglect the instantaneous friction term to give

$$
\frac{z \phi_{\mathbf{v}}(z)}{1+z \phi_{\mathbf{v}}(z)}=z m_{\mathbf{v}}(z)
$$

We now split the correlator into the sum of its late time limit and a (time-dependent) remainder, writing $\phi_{\mathbf{v}}(t)=f_{\mathbf{v}}+g_{\mathbf{v}}(t)$. Now we assume that, for suitable values of the relevant parameters, our model admits nonergodic solutions. In a nonergodic state the relaxation time $\tau$ is divergent and so $\dot{\gamma}=0$ for any finite stress. Therefore the zerofrequency limit of Eq. (14) gives

$$
\frac{f_{\mathbf{v}}}{1-f_{\mathbf{v}}}=V f_{\mathbf{v}}^{2}
$$

where $V \equiv v_{2}(\sigma)$. Here, we have used the fact that, since $g_{\mathbf{v}}(t)$ tends to zero at late times, terms of order $g_{\mathbf{v}}(t)$ or $g_{\mathbf{v}}^{2}(t)$ give rise to singularities weaker than $1 / z$ as $z$ $\rightarrow 0$. It has been shown [Götze and Sjögren (1995)] that the nonergodicity parameter $\left[\lim _{t \rightarrow \infty} \phi_{\mathbf{v}}(t)\right]$ is the largest of the solutions to Eq. (15).

This result fixes the nonergodicity parameter of an arrested state with coupling $V$ : there are nonzero solutions for $f_{\mathbf{v}}$ provided $V \geqslant 4$, so that a glass becomes possible beyond this value of the coupling. However, this does not imply that we necessarily have a glass transition at $V=4$, since we have calculated this by assuming arrest, which requires $\dot{\gamma}=0$. (Without shear, our model is simply a F2 model for which the result $V_{c}=4$ is well known.) In Sec. II, we stated that passing through a static MCT glass transition entails a discontinuity in the nonergodicity parameter $f$ followed by a nonanalytic variation as the coupling constants increase. Solving Eq. (15) shows that such behavior will arise in models I-IV only for an arrest transition occurring at the F2 value of $V=4$. We shall return to this point later. 


\section{Asymptotics for correlator and memory function}

We now consider the behavior of correlation functions close to a transition at (say) $\mathbf{v}_{c}$, corresponding to some $V>4$. Near such a transition, $\left|\mathbf{v}-\mathbf{v}_{c}\right|$ is a small quantity. To identify other quantities which may be treated perturbatively, we recall static MCT behavior: nonergodic solutions to Eq. (1) show a decay, at the $\beta$-time scale $\tau_{\beta}$, onto a plateau, with $\phi_{\mathbf{v}}\left(t>\tau_{\beta}\right) \approx f_{\mathbf{v}}$. For $V>4$, on time scales for which $\dot{\gamma}_{\mathbf{v}} t \ll 1$, the difference $\phi_{\mathbf{v}}(t)-\phi_{\mathbf{v}_{c}}(t)$ is also a small quantity. Provided $\dot{\gamma}_{\mathbf{v}}$ vanishes smoothly at the jamming transition, we can ensure that this condition holds at and beyond the time scale $\tau_{\beta}$ on which $\phi_{\mathbf{v}_{c}}(t)$ reaches its long time limit. That is, we choose $\left|\mathbf{v}-\mathbf{v}_{c}\right|$ such that $\dot{\gamma} \tau_{\beta} \ll 1$. (Our ability to do this depends on the fact that $\tau_{\beta}$ is finite, unlike $\tau_{\alpha}$, whenever $V>4$.) Thus we have identified a time regime where $g_{\mathbf{v}}(t) \equiv \phi_{\mathbf{v}}(t)-f_{\mathbf{v}_{c}}$ is a small quantity. In the frequency domain, this gives $z \phi_{\mathbf{v}}(z)=-f_{\mathbf{v}_{c}}+z g_{\mathbf{v}}(z)$. In line with standard MCT techniques [Götze (1989)], we now treat $g_{\mathbf{v}}(z)$ perturbatively in the Laplace transformed equation of motion, Eq. (14). Expanding the left-hand side in powers of $z g_{\mathbf{v}}(z)$, we find

$$
\frac{z \phi_{\mathbf{v}}(z)}{1+z \phi_{\mathbf{v}}(z)}=\frac{-f_{\mathbf{v}_{c}}}{1-f_{\mathbf{v}_{c}}}+\frac{z g_{\mathbf{v}}(z)}{\left(1-f_{\mathbf{v}_{c}}\right)^{2}}-\frac{z^{2} g_{\mathbf{v}}^{2}(z)}{\left(1-f_{\mathbf{v}_{c}}\right)^{3}}+\mathcal{O}\left[z^{3} g_{\mathbf{v}}^{3}(z)\right] .
$$

We now wish to approximate the right-hand side (rhs) of Eq. (14) in the same regime of frequency. By considering the memory function in the time domain, $\dot{\gamma}_{\mathbf{v}} t$ and $g_{\mathbf{v}}(t)$ can be treated perturbatively. So can the distance from the transition in parameter space: we write $V=V_{c}+\epsilon$ where $V_{c}$ is the value of $V \equiv v_{2}(\sigma)$ at $\mathbf{v}_{c}$.

To make further analytic progress we now restrict the form of the function $f(\dot{\gamma} t)$ in Eq. (4) to be exponential. (This excludes model IV; we have already excluded model V.) We find the memory function then to obey

$$
m_{\mathbf{v}}(t)=\left(V_{c}+\epsilon\right)\left[f_{\mathbf{v}_{c}}^{2}+2 f_{\mathbf{v}_{c}} g_{\mathbf{v}}(t)+g_{\mathbf{v}}^{2}(t)\right]\left[1-\dot{\gamma}_{\mathbf{v}} t+\mathcal{O}\left(\dot{\gamma}_{\mathbf{v}}^{2} t^{2}\right)\right] .
$$

In the time regime of interest, terms $\mathcal{O}\left[g_{\mathbf{v}}(t) \dot{\gamma}_{\mathbf{v}} t\right]$ and $\mathcal{O}\left[g_{\mathbf{v}}^{2}(t)\right]$ are negligible with respect to terms $\mathcal{O}\left[g_{\mathbf{v}}(t)\right]$. Additionally, terms $\mathcal{O}\left[\epsilon g_{\mathbf{v}}(t)\right]$ and $\mathcal{O}\left(\epsilon \dot{\gamma}_{\mathbf{v}} t\right)$ may be neglected with respect to terms $\mathcal{O}(\epsilon)$. The rhs of Eq. (14) then becomes

$$
z m_{\mathbf{v}}(z) \approx-V f_{\mathbf{v}_{c}}^{2}+2 f_{\mathbf{v}_{c}} V_{c} z g_{\mathbf{v}}(z)+V_{c} f_{\mathbf{v}_{c}}^{2} \dot{\gamma}_{\mathbf{v}} i / z
$$

Using Eq. (15) we find that close to the transition Eq. (14) becomes

$$
z g_{\mathbf{v}_{c}}(z)\left[\frac{1}{\left(1-f_{\mathbf{v}_{c}}\right)^{2}}-2 f_{\mathbf{v}_{c}} V_{c}\right]-V_{c} f_{\mathbf{v}_{c}}^{2} \dot{\gamma}_{\mathbf{v}} i / z+f_{\mathbf{v}_{c}}^{2} \boldsymbol{\epsilon}=0 .
$$

Inverting the Laplace transform, we find the correlator in the time domain to be

$$
\phi_{\mathbf{v}}(t) \approx f_{\mathbf{v}_{c}}\left[1+\frac{\epsilon f_{\mathbf{v}_{c}}}{\frac{1}{\left(1-f_{\mathbf{v}_{c}}\right)^{2}}-2 f_{\mathbf{v}_{c}} V_{c}}\right]\left[1-\frac{V_{c} f_{\mathbf{v}_{c}} \dot{\gamma}_{\mathbf{v}} t}{\frac{1}{\left(1-f_{\mathbf{v}_{c}}\right)^{2}}-2 f_{\mathbf{v}_{c}} V_{c}+\epsilon f_{\mathbf{v}_{c}}}\right],
$$

or, expressed more succinctly

$$
\phi_{\mathbf{v}}(t)=f(1-t / \tilde{\tau})
$$


with nonergodicity parameter $f$ and a relaxation time $\tilde{\tau}$ defined appropriately.

Equation (21) is consistent with an exponential decay from the plateau at late times, $\phi \sim f \exp (-t / \tilde{\tau})$, a result also found numerically in Sec. V later. In what follows we further assume that this is the correct late-time form for the correlator; this allows us to find explicitly the locus of arrest transitions arising in models I-III.

We begin by evaluating Eq. (13) at $z=0$ to deduce the existence of a terminal relaxation time

$$
\tau=\tau_{0}-i m(0)
$$

As we approach a jamming transition at finite stress, the relaxation time $\tilde{\tau}$ defined in Eq. (21) must diverge (since the shear rate vanishes at the transition point). We continue to assume that, at $\mathbf{v}_{c}, V>4$ in which case the $\tau_{\beta}$ remains finite. Therefore, approaching the transition, the terminal time $\tau$ is primarily set by the late-time exponential relaxation of $\phi_{\mathbf{v}}(t)$. Accordingly, Eq. (22) becomes

$$
\begin{aligned}
\tau & =\tau_{0}+V \int_{0}^{\infty} \exp (-\dot{\gamma} t) \phi^{2}(t) \mathrm{d} t \\
& \approx V \int_{0}^{\infty} \exp (-\dot{\gamma} t) f^{2} \exp (-2 t / \tilde{\tau}) \mathrm{d} t,
\end{aligned}
$$

where we have approximated the correlator by $\phi(t)=f \exp (-t / \tilde{\tau})$ over the whole temporal range (neglecting deviations at $t \lesssim \tau_{\beta}$ ), and dropped a (nondivergent) contribution from the regular part of the memory function. By definition, $\tau=\int_{0}^{\infty} \phi(t) \mathrm{d} t$, and so we require $\int_{0}^{\infty} f \exp (-t / \tilde{\tau})=\tau$, giving $\tilde{\tau}=\tau / f$.

We now write Eq. (24) as an iteration: the $n$th approximation to the relaxation time $\tau$ is calculated using the $(n-1)$ th value to determine the shear rate. Thus

$$
\tau^{(1)} \approx \int_{0}^{\infty} V f^{2} \mathrm{e}^{-\sigma t / \tau(0)} \mathrm{e}^{-2 f t / \tau(1)} \mathrm{d} t,
$$

which we can solve to give the ratio of $\tau^{(1)}$ to $\tau^{(0)}$ :

$$
\frac{\tau^{(1)}}{\tau^{(0)}}=\frac{f}{\sigma}(V f-2) .
$$

Crucially, this ratio is independent of $\tau^{(0)}$ : thus, no matter how large the initial guess, the iteration leads to a larger value if the rhs is greater than unity. Where this is the case, the relaxation time is divergent. Thus, setting the rhs in Eq. (26) to unity locates the arrest transition. Recalling the definition $V \equiv v_{2}(\sigma)$, this lies at a stress $\sigma_{c}$ which obeys

$$
f_{c}\left[v_{2}\left(\sigma_{c}\right) f_{c}-2\right]-\sigma_{c}=0,
$$

where $f_{c}$ is given by the largest solution of $f_{c} /\left(1-f_{c}\right)=v_{2}\left(\sigma_{c}\right) f_{c}^{2}$ [from Eq. (15)].

Except for the special case $\sigma_{c}=0$, these transition points do not coincide with the F2 transition at $V=4$, and have a different character to MCT transitions in general. From a rheological point of view, the transitions give values of the stress for which the shear rate first becomes zero: these are jamming transitions. Their locations according to Eq. (27) will later be checked against the numerical solutions for the full flow curves. 


\section{Large stress limit}

We turn now to investigate the behavior in the limit of large stress. We begin by considering cases in which the relaxation time diverges in that limit: in this case, Eq. (26) implies

$$
\lim _{\sigma \rightarrow \infty} \alpha f^{2} v_{2}(\sigma) / \sigma \geqslant 1 .
$$

The implications of this result depend upon the model: if $v_{2}(\sigma)$ increases sublinearly at large $\sigma$, this criterion will not be satisfied for any finite $\alpha$ and so fluid-like behavior is always recovered in the limit of large stress. For superlinear $v_{2}(\sigma)$, as arises in model II, this condition places no restriction upon $\alpha$ beyond $\alpha>0$. Hence, model II always has zero shear rate at high stresses: the jammed state remains solid indefinitely.

The most interesting case is when $v_{2}(\sigma)$ increases linearly at large $\sigma$, which applies in both models I and III. Here Eq. (28) can only be satisfied if $\alpha \geqslant 1$, since in a jammed state the nonergodicity parameter $f$ will approach unity in the limit of large stress [see Eq. (15)]. This implies that, for $\alpha<1$, the shear rate can be made arbitrarily large by increasing the stress. In this case, we can calculate the relaxation time in the large stress limit from Eq. (23). Only the region $t \sim 1 / \dot{\gamma}$ contributes significantly to the integral, and for large shear rates this region becomes arbitrarily small. Thus, we may expand the correlator in powers of $t$ [Hinch (1991)], giving

$$
\int_{0}^{\infty} \mathrm{e}^{-\dot{\gamma} t} \phi^{2}(t) \mathrm{d} t \approx \int_{0}^{\infty} \mathrm{e}^{-\dot{\gamma} t}\left[\phi^{2}(0)+\mathcal{O}(t)\right] \mathrm{d} t .
$$

The initial condition $\phi(0)=1$ (normalization) gives

$$
\tau \approx \tau_{0}+v_{2}(\sigma) \int_{0}^{\infty} \mathrm{e}^{-\sigma t / \tau} \mathrm{d} t
$$

which is solved in the limit of large stress by

$$
\tau=\frac{\tau_{0}}{1-\alpha} \quad(\alpha<1)
$$

If $\tau$ grows sublinearly with $\sigma$ at large stress, both quantities diverge in this limit. In this case $\alpha \geqslant 1$ and $\tau=\tau_{0} /(1-\alpha)$; demanding a non-negative relaxation time leads to the conclusion that $\alpha=1$. Thus, in model I, $\alpha=1$ is a watershed between ergodic and nonergodic solutions in the limit of large stress. For $\alpha<1$, solutions are ergodic with the viscosity approaching a limiting value; but for $\alpha>1$, the flow rate $\dot{\gamma}$ is zero at high stress.

\section{B. Numerical solutions}

The preceding analytical results are useful but restricted to certain model variants. Also they do not provide closed form expressions for flow curves (only transition points). We have therefore also solved our schematic rheological models numerically, using an established algorithm for MCT models [Fuchs et al. (1991)]. The numerical method involves rewriting the governing equation in such a way that $\phi(t)$ is determined from its value at earlier times, allowing iterative calculation of correlation functions. Numerical efficiency is aided by treating slowly varying quantities as constant over the time step. To adapt this algorithm to our models, we employ the following procedure: 
(1) Input the parameters of interest;

(2) Make an initial guess at the relaxation time $\tau$ for these parameters;

(3) Calculate the shear rate using this relaxation time and the chosen value of the stress. This defines iteration-dependent coupling constants $v_{n}(\sigma)$ and the memory loss function $f(\dot{\gamma} t)$;

(4) Solve the resulting model via the methods of Fuchs et al. (1991) (holding the coupling constants fixed);

(5) Recalculate the relaxation time by integrating the resulting correlator; and

(6) Repeat from step 3 onwards using the new value of the relaxation time until converged. The result is a self-consistent solution.

Numerical solutions using this algorithm were obtained using a FORTRAN code. We checked that the code returned the expected F2, F12 results for $\sigma=0$. For some parameters close to a nonergodicity transition, the code became slow to converge, especially for "bad" initial guesses. This problem was circumvented by starting the iteration off with a range of initial values and bracketing the correct value according to whether the initial trajectories showed increasing or decreasing $\tau$. In some cases this generated improved initial values from which the earlier algorithm could attain convergence; in others, the process was simply continued until converged to the required accuracy in its own right. (The stability of results obtained in this manner was then checked by a final iteration.)

\section{RESULTS: MODEL I}

In this section we present detailed results for model I. Later (Sec. VI) we shall examine the effect of altering the form of the memory function, resulting in models II-V. (This is easier than attempting to present results for all the model variants in parallel; it does not necessarily mean that we prefer model I to the rest.) From now on we use units where the local relaxation time far from the glass transition, $\tau_{0}$, is unity. We continue to set the transient elastic modulus $G$ to unity and can therefore identify the viscosity $\eta$ with the terminal relaxation time $\tau$.

\section{A. Steady-state rheology}

\section{Choice of parameters}

We expect the most interesting behavior to arise close to the quiescent stress-free glass transition, where there is maximal interplay between the long relaxation times and the effect of shearing. For hard-sphere colloids, discontinuous thickening tends to occur for concentrations $\phi \geqslant 0.5$, for which the zero-shear viscosity is around 50 times that of the solvent [Meeker et al. (1997)]. Thus, we first study values of $v_{2}$ for which $\tau \gtrsim 50$ (recall that $\tau_{0}=1$ ). The stress-coupling parameter $\alpha$ does not have such an obvious experimental analog, and it is less clear what values to investigate. However, from Sec. IV, we expect values of order unity to lead to stress-induced nonergodicity, so we mainly consider such values. (As indicated in Sec. IV A 2, and confirmed below, $\alpha=1$ in fact separates differing regimes of behavior.) Stress can result in thickening, in principle, whenever $v_{2}+\alpha \sigma \simeq 4$ : but whether this actually occurs depends on the interplay with shearing-induced memory loss.

\section{Dependence on $v_{2}$}

We begin by varying $v_{2}$ at a fixed value of $\alpha$, chosen slightly less than unity. Figure 1 shows the flow curves for $\alpha=0.95$ with $3.5 \leqslant v_{2} \leqslant 3.8$. In all cases, there is a regime of shear thinning and, for the largest value of $v_{2}$, the shear thinning is preceeded by 


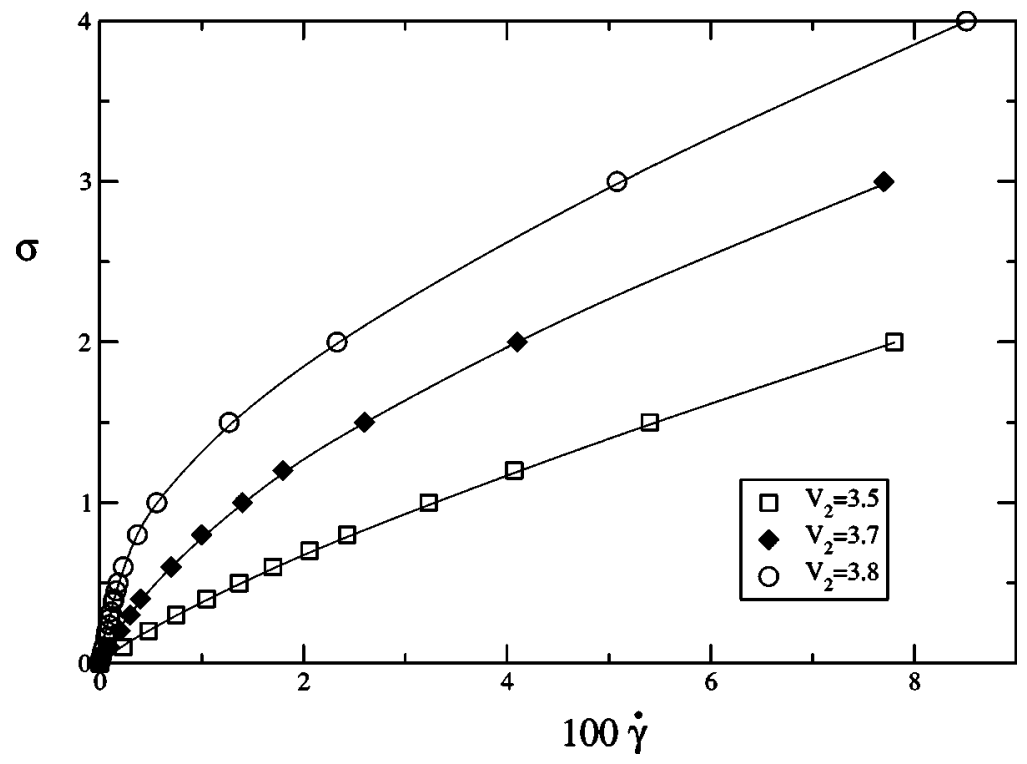

FIG. 1. Model I flow curves for $\alpha=0.95$ : points are numerically determined values of the shear rate for a given stress. Shear thinning is seen and, for $v_{2}=3.8$, thickening is also discernable at low stresses.

thickening at small applied stresses. In all flow curves presented (here and below) lines are to guide the eye. For more modest values of $v_{2}$ the rheology, not shown, is close to Newtonian. (For $v_{2}=3$, for example, the viscosity changes only by about $15 \%$ upon increasing the stress between zero and $\sigma=5$.)

Figure 2 shows how the trend towards shear thickening develops upon further increasing $v_{2}$. In contrast to the rather gentle curves of Fig. 1, the behavior observed here is rather drastic. As $v_{2}$ is increased above 3.8, the shear thinning behavior shown in Fig. 1 is interrupted by shear thickening whose severity ranges from modest to extreme-for the larger values of $v_{2}$ shown, the thickening becomes so strong that regions of negative slope appear. In these regions, increasing the stress lowers the shear rate. Such regions of negative slope should be mechanically unstable to shear banding [Dhont (1999), Olmsted (1999)] and should lead in practice to discontinuous shear thickening as we discuss in Sec. VII.

For the largest values of $v_{2}$ shown, there is a window of stress for which the viscosity has diverged, resulting in "full jamming" - the creation of a nonflowing state by application of stress [Hess and Hess (1994), Holmes et al. (2003)]. Within this window, the only solution is a nonergodic, jammed state. The edges of this window are the jamming transitions whose positions are given analytically by Eq. (27). The analytic and numerical results for their positions are in agreement. We examine in more detail the nature of these transitions in Sec. VII.

The scenario describes the stress induced arrest of a state with $v_{2}<4$; this is a liquid at low stresses and therefore the region of the flow curve close to the origin is always Newtonian (albeit with a viscosity that diverges as $v_{2} \rightarrow 4$ ). By setting $v_{2} \geqslant 4$, we can consider shearing a system that is arrested in the quiescent state (the usual definition of a colloidal glass). In this case, as shown in Fig. 3, there is no steady-state flow unless the stress is increased beyond a yield stress which itself increases monotonically with $v_{2}$. (No new features arise at larger $v_{2}$ than those shown here.) The application of stresses 


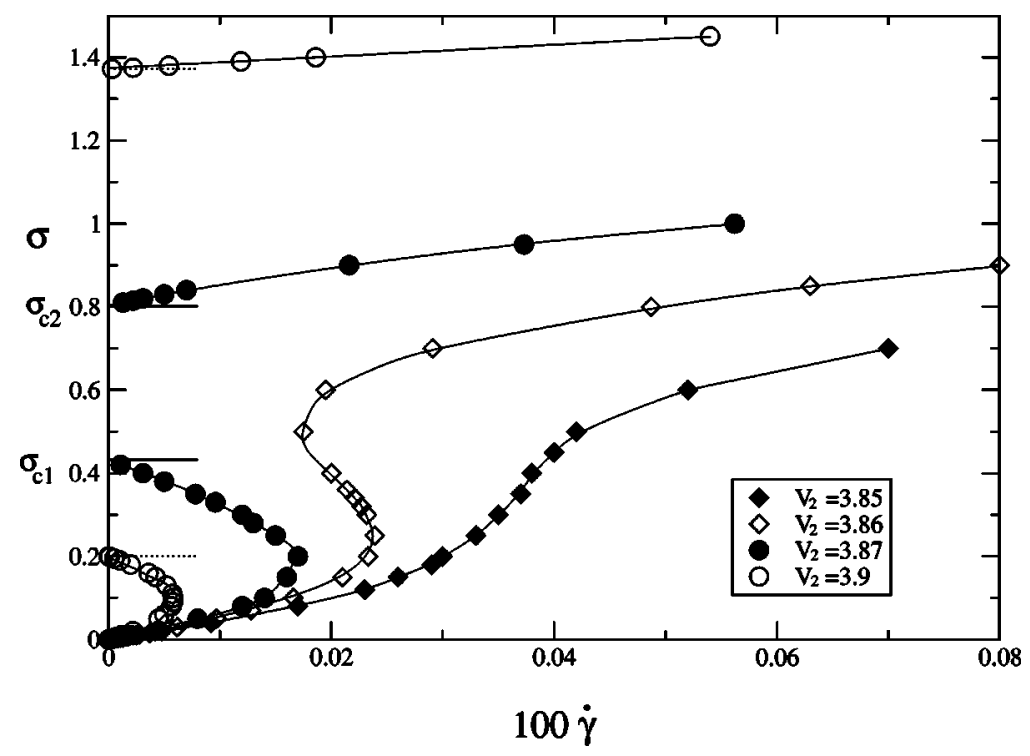

FIG. 2. Further model I flow curves for $\alpha=0.95$. For the two largest values of $v_{2}$, there is a window of stress for which the viscosity diverges. The limits of this window (denoted $\sigma_{c 1}$ and $\sigma_{c 2}$, as shown here for $v_{2}$ $=3.87$ ), are jamming transitions. The predicted values of these, according to Eq. (27), are shown as horizontal line segments near the stress axis.

beyond the yield stress lead to 'shear melting' of the glass, followed by a thinning viscosity, similar to the behavior at stresses above $\sigma_{c 2}$ in Fig. 2.

Our results for model I with $\alpha=0.95$ can then be summarized as follows: far from the quiescent glass transition $\left(v_{2} \lesssim 3\right)$, the rheology is quasi-Newtonian; for $3 \lessgtr v_{2}$

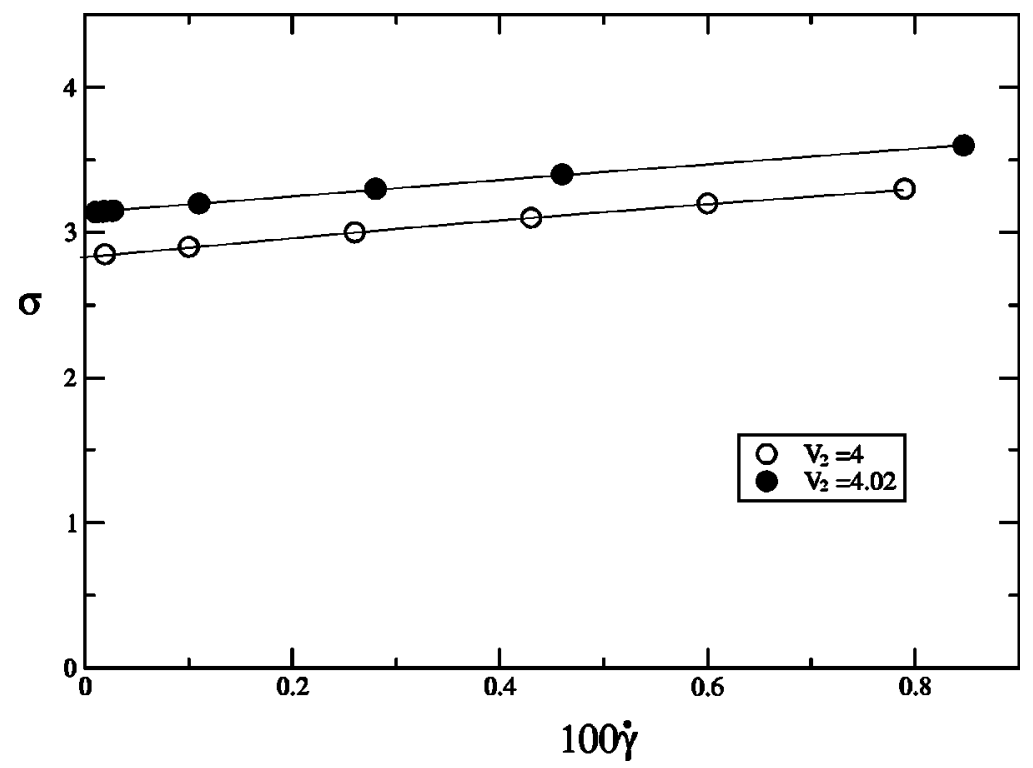

FIG. 3. Model I flow curves for $\alpha=0.95$, with $v_{2} \geqslant v_{2}^{c}=4$. Steady state flow occurs only for stresses above the yield stress. 


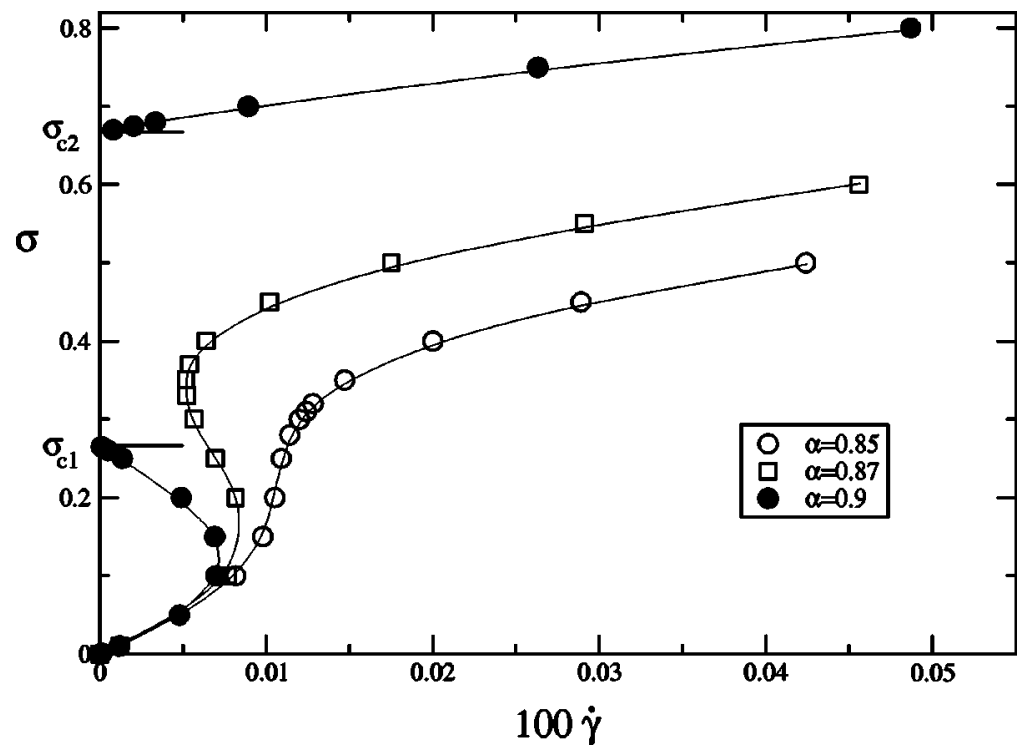

FIG. 4. Model I flow curves at $v_{2}=3.9$ for various $\alpha$. For $\alpha=0.9$, jamming transitions and their analytical locations are indicated as in Fig. 2.

$\lesssim 3.8$, shear thinning is apparent. In the range $3.8 \lesssim v_{2}<4$, shear thickening becomes prominent: upon increasing $v_{2}$ the flow curve first becomes nonmonotonic, and then (for $v_{2} \gtrsim 3.866$ ) the curve reaches all the way back to the stress axis, indicating a jamming transition to a nonergodic state. Finally, for $v_{2} \geqslant 4$, a conventional yield stress appears, below which there is no (steady-state) flow. At stresses exceeding this yield stress, behavior is similar to that above $\sigma_{c 2}$ in the full jamming flow curves.

\section{Dependence on $\alpha$}

We now fix the value of $v_{2}$ and investigate the effect of varying $\alpha$. As mentioned earlier, the experimental meaning of $\alpha$ is not obvious: it controls the extent to which the mode coupling vertex increases with applied stress, and seems related to the susceptibility of the static structure to external forcing. Here we choose $v_{2}=3.9$ (for which the zero-shear viscosity is $\tau \approx 814$ ): this is close enough to the quiescent glass transition to uncover some interesting behavior. This behavior arises for $\alpha$ values of order unity; note that if instead $\alpha \ll 1$ there is no shear thickening. Flow curves for a range of $\alpha \lesssim 1$ are shown in Fig. 4. The variation in the curves with increasing $\alpha$ is qualitatively similar to that in Fig. 2, with the shear thickening becoming stronger as $\alpha$ is increased.

In fact there is an important qualitative distinction between Figs. 4 and 2 which is not immediately apparent from the plots. Specifically, as $\alpha$ is varied, the asymptotic slope of the flow curves at large stresses diverges as $\tau \sim 1 /(1-\alpha)$ (Sec. IV A 2). In contrast, varying $v_{2}$ at constant $\alpha<1$ leads always to curves of the same (finite) limiting slope. For $\alpha>1$, the slope of the flow curve at large stresses remains infinite which means there is no upper branch at large $\dot{\gamma}$ as illustrated in Fig. 5. This is consistent with our earlier conclusion that, for $\alpha>1, \lim _{\sigma \rightarrow \infty} \tau=\infty$. However, the topology of Fig. 5 is not the only possibility: there is a small region of parameter space $(1<\alpha \leq 1.0032$ for $\left.v_{2}=3.9\right)$ for which the system refluidises upon increasing the stress, followed by a 


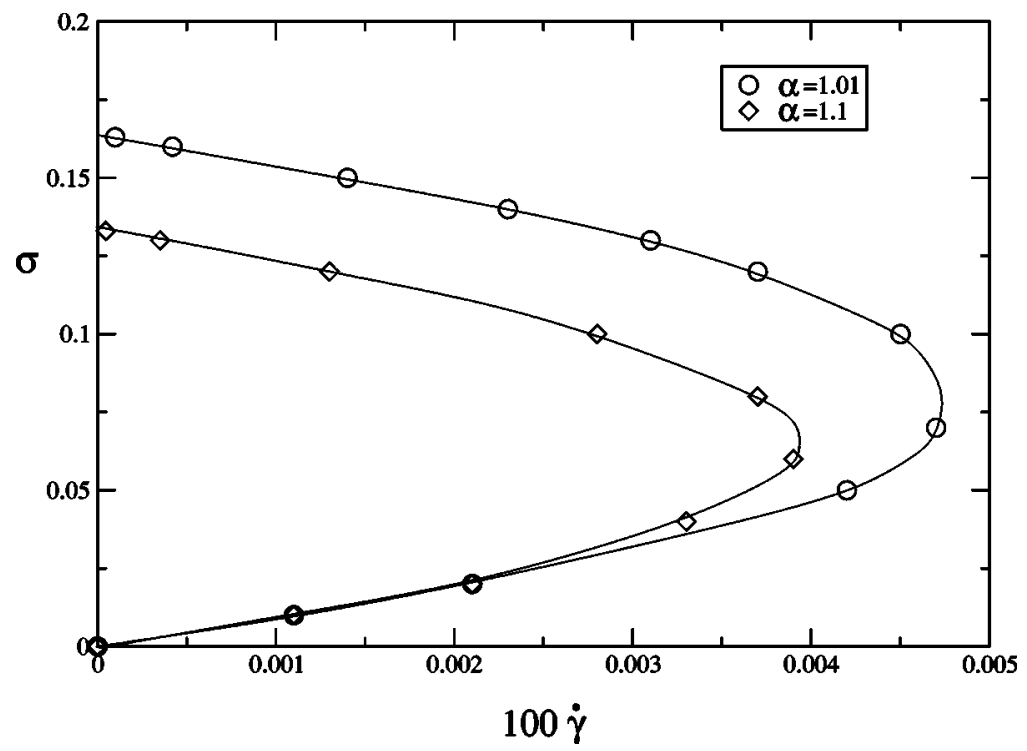

FIG. 5. Model I flow curves at $v_{2}=3.9$ with $\alpha>1$. There is no refluidisation from the jammed state to a high stress fluid.

second window of nonergodicity, which persists indefinitely upon further increasing $\sigma$. This somewhat peculiar behavior is illustrated in Fig. 6.

A remark is in order on the physical meaning of flow curves that show no liquid-like branch at high stresses. We do not expect that applying an arbitrarily large stress will fail to make a colloidal system flow at all; but the flow could be unsteady, or could be spatially nonuniform involving a fracture mechanism, for example. Neither outcome is

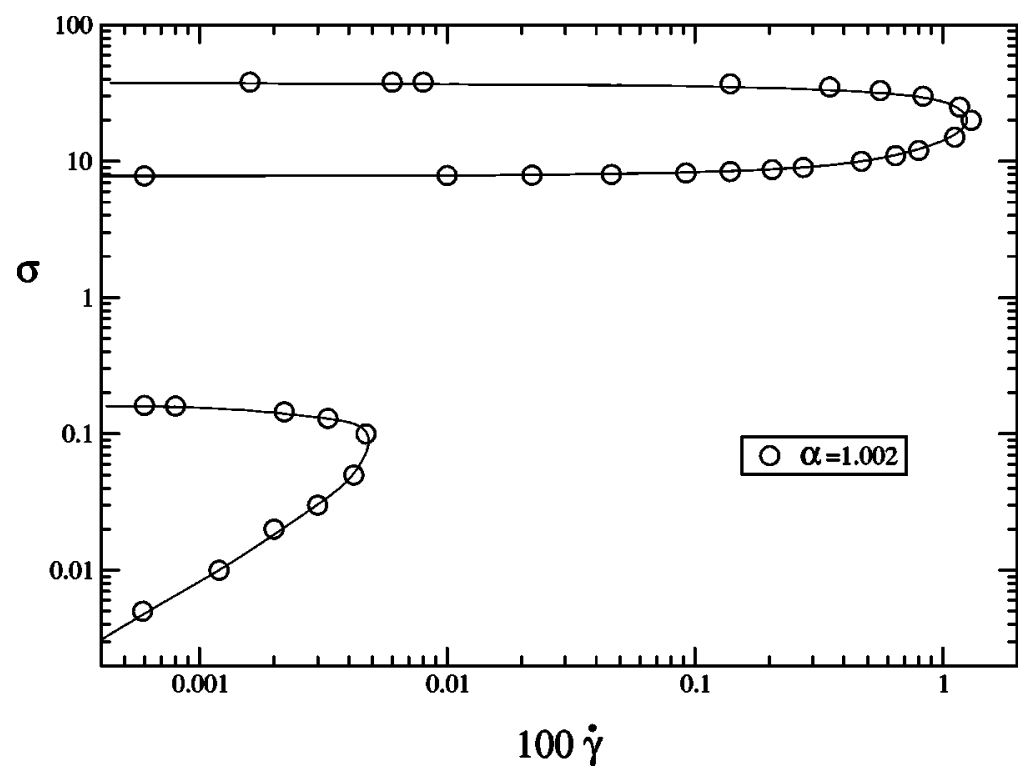

FIG. 6. Model I flow curve for $\alpha=1.002$ (in a $\log -\log$ plot), showing two windows of jamming. Fluidization from the second window by increasing the stress is not possible. 


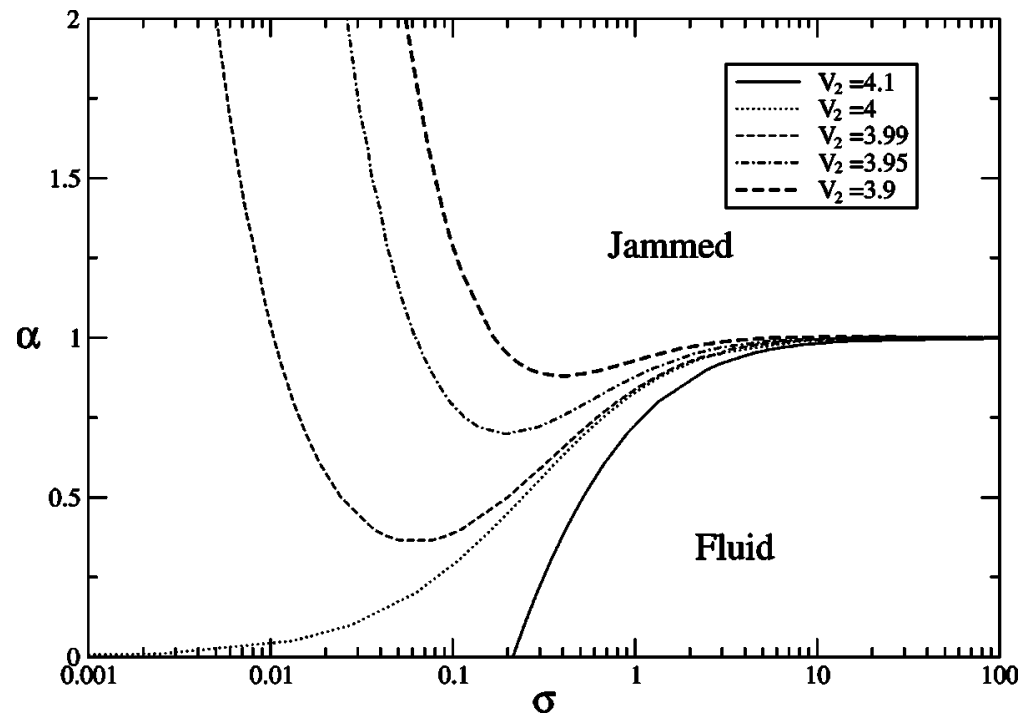

FIG. 7. Phase diagrams for model I at various $v_{2}$. The curves denote jamming transitions. All states below the curve for a given value of $v_{2}$ are fluid states, while those above (and on) the line are nonergodic, jammed states.

addressable within the modeling framework developed here, so that flow curves such as those of Fig. 5 need not be regarded as unphysical in our context. On the other hand, whenever $\alpha>1$ such flow curves arise even for small $v_{2}$, far from the quiescent glass transition. This would surely be unphysical so we assume that if $\alpha$ ever exceeds 1 , it does this only at high concentrations.

A final, intermediate flow scenario is found at the critical value $\alpha=1$. In this case it is difficult to resolve some important details of the flow curve numerically: there is nontrivial structure in regions where $\sigma$ and $\tau$ are large. There is certainly a "low stress" window of nonergodicity for $0.165 \lesssim \sigma \lesssim 5.935$ (for $v_{2}=3.9$ ). Upon increasing the stress further, the jammed state yields, followed by a second regime of thickening in which $\tau$ becomes too large to track numerically. This suggests that a second nonergodicity transition might occur: however, we shall see in Sec. V B [by examining Eq. (27)] that this is not the case.

\section{B. Phase diagrams}

This concludes our survey of flow curves for model I, which has covered the basic rheological scenarios found. To get an overview of the behavior over a wider range of parameters, we now concentrate on the jamming transitions and their locations in parameter space. By solution of Eq. (27) for a range of $v_{2}$ and $\alpha$, we can calculate "phase diagrams" depicting the boundary in parameter space between ergodic and nonergodic regions.

\section{The $(\sigma, \alpha)$ representation}

Phase diagrams of model I, in the $(\sigma, \alpha)$-plane, are shown in Figs. 7 and 8. This representation of the phase behavior shows that, at a given value of $v_{2}$, nonergodic states appear provided $\alpha$ is sufficiently large. As $v_{2}$ is lowered below 4 (so that the system at rest is ergodic), the minimum value of $\alpha$ necessary for nonergodicity under stress increases from zero until, for $v_{2}$ less than a critical value $\tilde{v}_{2}$ (found later) there is no 


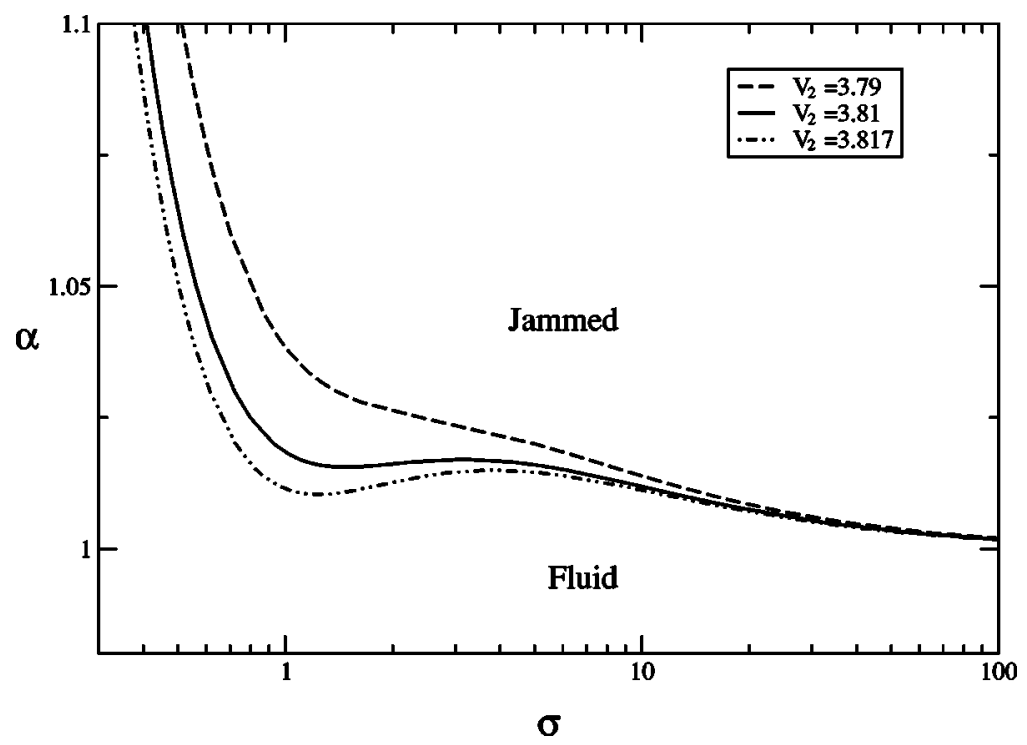

FIG. 8. Phase diagrams for model I at smaller $v_{2}$. (Note the different scale to Fig. 7.) As before, jammed states lie on or above the line for a given value of $v_{2}$.

transition unless $\alpha>1$. Once this condition applies, a transition occurs regardless of any further decrease of $v_{2}$ [Eq. (28)].

In Fig. 4 (respectively, Fig. 5), we showed examples of model I flow curves in which a jammed state could (could not) be refluidized by further increasing the stress. Figures 7 and 8 show that such refluidization occurs within a window of $\alpha$ which disappears when $v_{2}$ falls below a second critical value $\tilde{\tilde{v}}_{2}$ (also found later). Outside of this window, the upper, refluidized branch of the flow curve is absent (Fig. 5). When such a window of $\alpha$ is present at $\alpha>1$ (visible for the lower two curves in Fig. 8) an upper branch of the flow curve exists, but its relaxation time diverges in the large stress limit and so this branch shows a second jamming transition with no ultimate fluidization at high stress (as in Fig. 6). Flow curves showing full jamming with a single refluidized branch (as visible in Figs. 2 and 4) thus require both $\alpha$ somewhat less than 1 and $v_{2}$ somewhat less than 4.

\section{The $(\sigma, V)$ representation}

For any given parameter set, the $(\sigma, \alpha)$ representation of the phase diagram provides a useful picture of the behavior under increasing the stress. However, the full range of jamming scenarios is most easily summarized using a different representation. This involves plotting the locus of transitions in the $(\sigma, V)$ plane, where we recall that $V$ $\equiv v_{2}(\sigma)$. We refer to the resulting transition curve as $V_{c}(\sigma)$; it is found by solving Eq. (27) for $v_{2}$ at fixed $\sigma=\sigma_{c}$, and has no dependence on parameters such as $\alpha$. Now, in model I, $V(\sigma)$ is just a straight line with slope $\alpha$ which intercepts the $V$ axis at $v_{2}$. Each transition point, for given $v_{2}$ and $\alpha$, corresponds to an intersection of $V(\sigma)$ with $V_{c}(\sigma)$. The range of behavior obtained upon varying $v_{2}$ and $\alpha$ simply corresponds to the different ways in which a straight line may intersect the fixed locus of possible transition points represented by the curve $V_{c}(\sigma)$. This is illustrated in Fig. 9 where, in the main figure, the dotted line corresponds to $v_{2}=3.9, \alpha=0.95$ : the two intersections of the transition boundary correspond to jamming, followed by refluidization. The dashed line (corresponding to $v_{2}=3.8, \alpha=1.1$ ) has a single intersection, showing that for these param- 


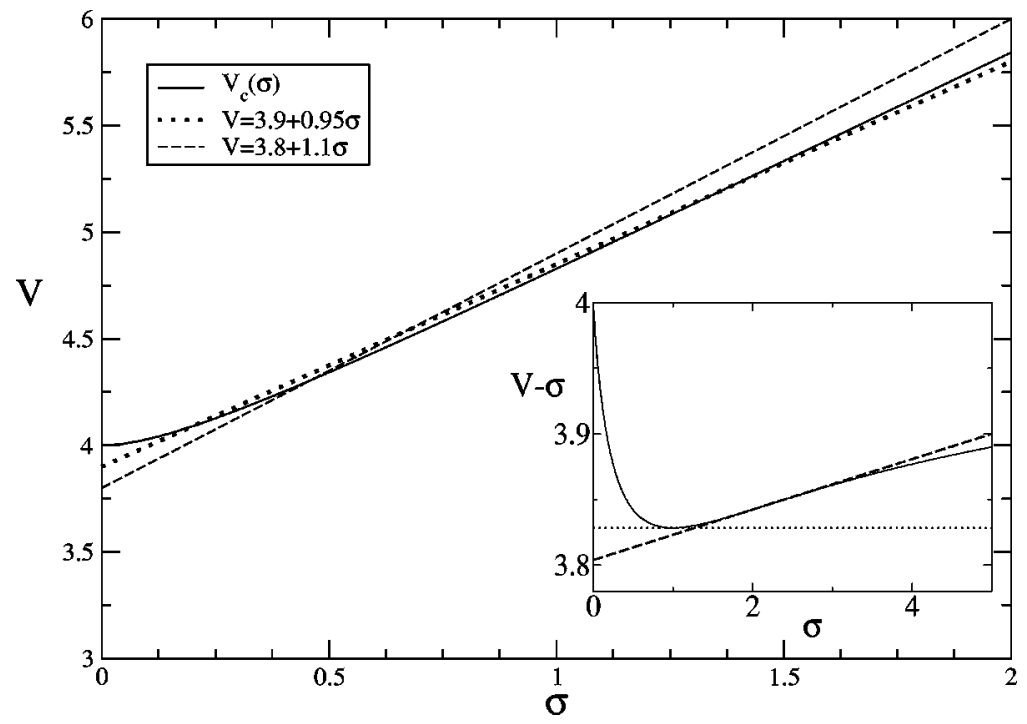

FIG. 9. Phase diagram in the $(\sigma, V)$-plane. Above and on the transition boundary (solid line) states are nonergodic. Inset: $V_{c}(\sigma)-\sigma$ (solid line) as a function of $\sigma$.

eters there is no refluidization of the jammed state. In order to bring out the structure present in $V_{c}(\sigma)$, in the inset we plot $V_{c}(\sigma)-\sigma$ (solid line) as a function of $\sigma$. We show the tangent to this curve at its point of maximum slope (dashed line), whose intercept with the vertical axis identifies $\tilde{\tilde{v}}_{2}$ as explained later. For $\alpha=1$ the line $V(\sigma)-\sigma$ is independent of $\sigma$ and has the value $v_{2}$. Below we identify $\tilde{v}_{2}$ as the smallest value taken by $V_{c}(\sigma)-\sigma$ : the dotted line corresponds to $V-\sigma=\tilde{v}_{2}$.

\section{Implications for $\tilde{v}_{2}, \tilde{\mathbf{v}}_{\mathbf{2}}$}

Earlier, we stated that, for $v_{2}<\tilde{\tilde{v}}_{2}$ multiple transitions are no longer allowed. The $(\sigma, V)$ representation allows a precise calculation of $\tilde{\tilde{v}}_{2}$, as follows. From Eq. (27), the maximum slope of $V_{c}(\sigma)$ is $V_{c}^{\prime}\left(\sigma_{\max }\right)=27-15 \sqrt{3} \approx 1.019$ at $\sigma_{\max }=1+2 / \sqrt{3}$. For $\alpha \geqslant V_{c}^{\prime}\left(\sigma_{\max }\right)$, there is then a single jamming transition if $v_{2}<4$. For refluidization to occur, the jamming transition must occur at a stress $\sigma<\sigma_{\max }$ : beyond this point the slope $V_{c}^{\prime}(\sigma)$ is monotonically decreasing and so a straight line cutting the transition curve (from below) beyond $\sigma_{\max }$ cannot encounter it subsequently. The critical value $\tilde{\tilde{v}}_{2}$ is then found by setting $v_{2}+V_{c}^{\prime}\left(\sigma_{\max }\right) \sigma_{\max }=V_{c}\left(\sigma_{\max }\right)$; that is, $\tilde{\tilde{v}}_{2}$ is the intercept on the $V$ axis of the tangent to the point of maximum slope. This gives $\tilde{v}_{2}=9(1-1 / \sqrt{3})$ $\approx 3.804$. This geometrical interpretation is illustrated in the inset to Fig. 9 .

The slope of the transition line $V_{c}(\sigma)$ approaches unity at large stresses ensuring that, for $\alpha>1$, the system is nonergodic in the limit. In contrast, for $\alpha<1$, jamming transitions occur only if $v_{2}$ is sufficiently large. The case $\alpha=1$ is of particular interest, as we were unable to elucidate its behavior numerically. The minimum value of $v_{2}$ for which a transition occurs (with $\alpha=1$ ) is $\tilde{v}_{2}$, as defined earlier. This is now obtained by asking what is the minimum intercept (with the $V$ axis) for which a straight line of unit slope cuts the transition line $V_{c}(\sigma)$. This yields a value of $\tilde{v}_{2}=1+2 \sqrt{2} \approx 3.828$. (Again, the geometrical interpretation, suitably altered, is illustrated in the inset to Fig. 9.) At $\alpha=1$, there is no transition below this value of $v_{2}$; for $\tilde{v}_{2}<v_{2}<4$ there are two transitions 


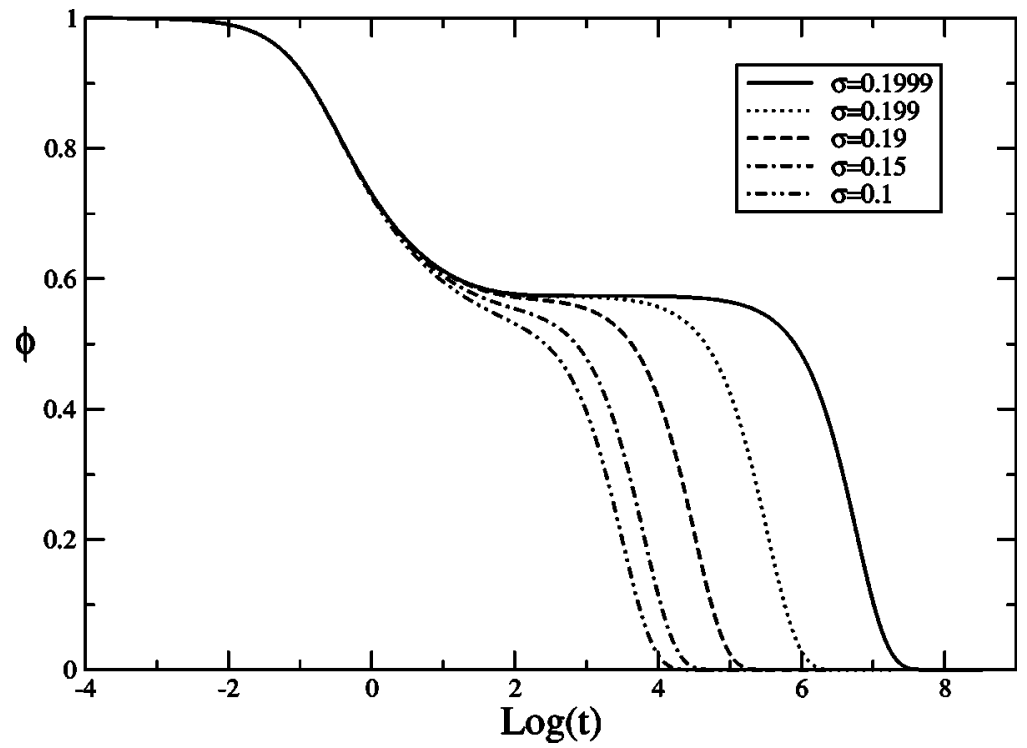

FIG. 10. Model I correlation functions for stresses approaching $\sigma_{c 1}=0.2$ for $v_{2}=3.9, \alpha=0.95$. Sufficiently close to the transition, there is a clear separation between the $\beta$-decay and the flow induced final decay, resulting in a well defined plateau.

(jamming and refluidization at $\sigma_{c 1}$ and $\sigma_{c 2}$, respectively). As $v_{2} \rightarrow(1+2 \sqrt{2})^{+}, \sigma_{c 1}$ and $\sigma_{c 2}$ merge, and as $v_{2} \rightarrow 4^{-}, \sigma_{c 1} \rightarrow 0$ and $\sigma_{c 2} \rightarrow \infty$.

We have shown that our critical values of $v_{2}$ obey $\tilde{v}_{2}>\tilde{v}_{2}$. For $v_{2}$ between these two values, flow curves with two separate jammed states (Fig. 6) can be found, but there are no flow curves with a single jammed state refluidised at high stress, as seen in Figs. 2 or 4.

\section{Yielding of a quiescent glass}

Finally, this representation of the phase diagram allows us to elucidate the yielding of a quiescent glass within model I. As $\sigma \rightarrow 0, V_{c}(\sigma) \rightarrow 4$, and $V_{c}^{\prime}(\sigma) \rightarrow 0$. Therefore, for $\alpha=0$, the yield stress is zero at the quiescent transition $\left(v_{2}=4\right)$ and increases smoothly upon moving deeper into the glass. This differs from the microscopic MCT calculations of Fuchs and Cates $(2002,2003)$ where, although there is no explicit stress dependence of the vertex, the yield stress rises discontinuously from zero at the static glass transition. However, in model I any $\alpha$ obeying $0<\alpha<1$ restores this expected discontinuity of yield stress at the static F2 transition. The resulting yield stress increases with $\alpha$ and diverges for $\alpha \geqslant 1$, beyond which, within model I, the glass cannot be shear melted at any finite stress.

\section{Relaxational dynamics}

The results presented thus far are determined solely by the relaxation time (equivalently, viscosity) for given parameter sets. This is the time integral of the correlator. We now consider the functional form, rather than just the time integral, of the correlator $\phi(t)$ in model I.

Figure 10 shows the correlation functions on approaching a typical jamming transition. At early times the correlators for a range of applied stress are very similar: the $\beta$-relaxation time scale (on which the initial decay of correlations occurs) is approxi- 


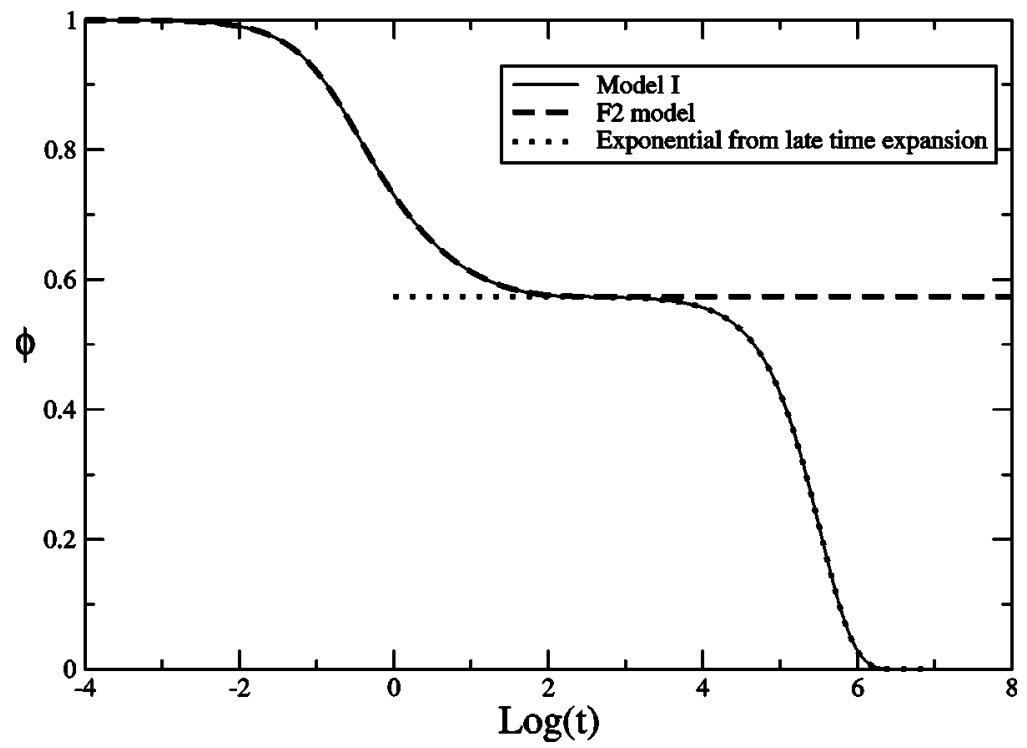

FIG. 11. The correlation functions for model I with $v_{2}=3.9, \alpha=0.95$ and $\sigma=0.199$, compared with the correlation function for the F2 model with $v_{2}=3.9+(0.95 \times 0.199)=4.08905$, and (at late times) with the exponential consistent with Eq. (20). At $\sigma=0.199$, the shear rate is $\dot{\gamma}=1.04 \times 10^{-6}$ (the initial jamming transition for these parameters is at $\sigma_{c 1}=0.2$ ).

mately constant. The correlators differ significantly only in their terminal relaxation times $\tau \sim 1 / \dot{\gamma}$. This shear-induced relaxation time diverges as the jamming transition is approached $(\dot{\gamma} \rightarrow 0)$. The consequences of this are as discussed in Sec. IV A. On time scales $t \ll 1 / \dot{\gamma}$, model I behaves as a static F2 model with coupling constant $V \equiv v_{2}$ $+\alpha \sigma$ which, unless $V=4$, has a finite $\tau_{\beta}$. Figure 11 illustrates the equivalence of correlations in model I, at times $t \ll 1 / \dot{\gamma}$, with the "underlying," noncritical, F2 model.

In calculating the whereabouts of transition points (Sec. IV A) we assumed that, in the vicinity of a jamming transition, correlation functions were well described by an exponential at late times, and cited numerical evidence. Figure 11 demonstrates this for one particular parameter set. A similar agreement has been found near jamming transitions for other parameters, including transitions at both $\sigma_{c 1}$ and $\sigma_{c 2}$.

\section{MODELS II-V}

Having studied model I in some detail, we turn now to consider results from models II-V, focusing on qualitative differences between these and model I. By doing so, we hope to clarify which results depend on particular choices made in setting up that model, and which are more robust.

\section{A. Model II}

The analytic work of Sec. IV A applies to model II, and so the phase behavior is again determined via Eq. (27). [As with model I, numerics show that the late-time decay of correlations is well described by an exponential, verifying the analysis leading to Eq. (27).] Using the $(\sigma, V)$ representation of the phase diagram we can elucidate the phase behavior of this model. The transition line $V_{c}(\sigma)$ remains unchanged from model I, whereas $V(\sigma)$ is now quadratically increasing with stress. Since the maximum slope of $V_{c}(\sigma)$ is finite, for sufficiently large stresses $V(\sigma)>V_{c}(\sigma)$ and so, provided only that 


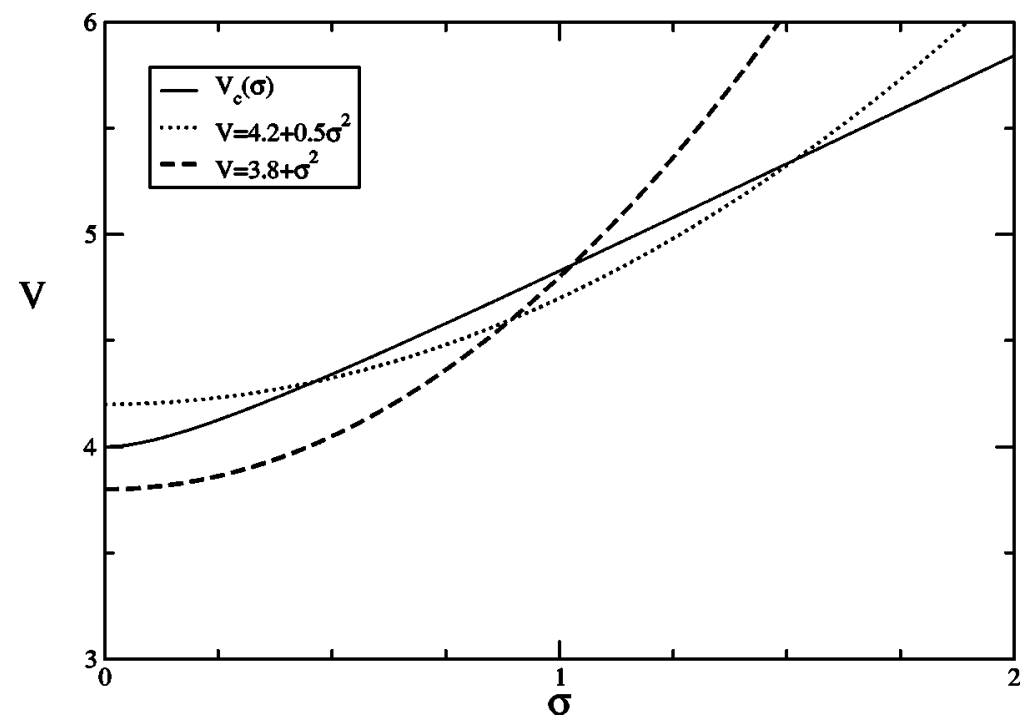

FIG. 12. The $(\sigma, V)$ representation of the phase diagram for model II. As in Fig. 9, intersections of the transition line $V_{c}(\sigma)$ with $V(\sigma)$ mark the jamming transitions. The quadratic stress dependence of $V(\sigma)$ in model II ensures at least one jamming transition. At high enough stress, model II is nonergodic, although (as shown) a static glass may be shear melted before jamming.

$\alpha>0$, model II exhibits a jamming transition irrespective of other parameters. This ensures that, in the limit of high stress, model II is nonergodic. Stress-induced jamming is never followed by refluidisation: and while a quiescent glass may be shear melted (depending on $\alpha$ ), this will always jam again at higher stresses. These scenarios are illustrated in Fig. 12. We have obtained flow curves (not shown) corresponding to both of these scenarios.

The flow curves of model II also differ from model I at low stresses for a system that is liquid at rest; here one finds (for suitable parameters) a region of downward curvature near the origin. Hence, there is an initial shear thinning region before the shear thickening and arrest scenarios are encountered. (As far as we know, this is generally the case experimentally.) Figure 13 shows the flow curves for model II with $v_{2}=3.9, \alpha=0.2$, for which full jamming is present: compare this to the flow curves for $v_{2}=3.9$ in model I (Fig. 4), in which no thinning is apparent prior to thickening.

\section{B. Model III}

We have just seen that in model II the system always jams at high stresses but is shear thinning at low ones. In Sec. III we argued that, around $\sigma=0$, the quadratic form (model II) has a sounder basis $\left[v_{2}(\sigma)\right.$ should be a symmetric function] whereas there is no reason to restrict attention to a quadratic at larger stresses. The wider variety of flow scenarios emerging from model I at high stresses suggests some utility for a model in which a quadratic stress dependence at low stresses goes over to a less severe form at higher ones. Model III [see Eq. (9)] provides a simple way to allow for this.

As shown in Fig. 14, in model III shear thinning can preceed thickening, just as in model II. Unlike model II, however, full jamming may now be followed by refluidization: the high stress limit is ergodic if $\alpha$ is smaller than unity. This is illustrated by the phase diagram trajectory shown in Fig. 15, in which a quiescent glass is first fluidized, then jams, and is finally refluidized again. 


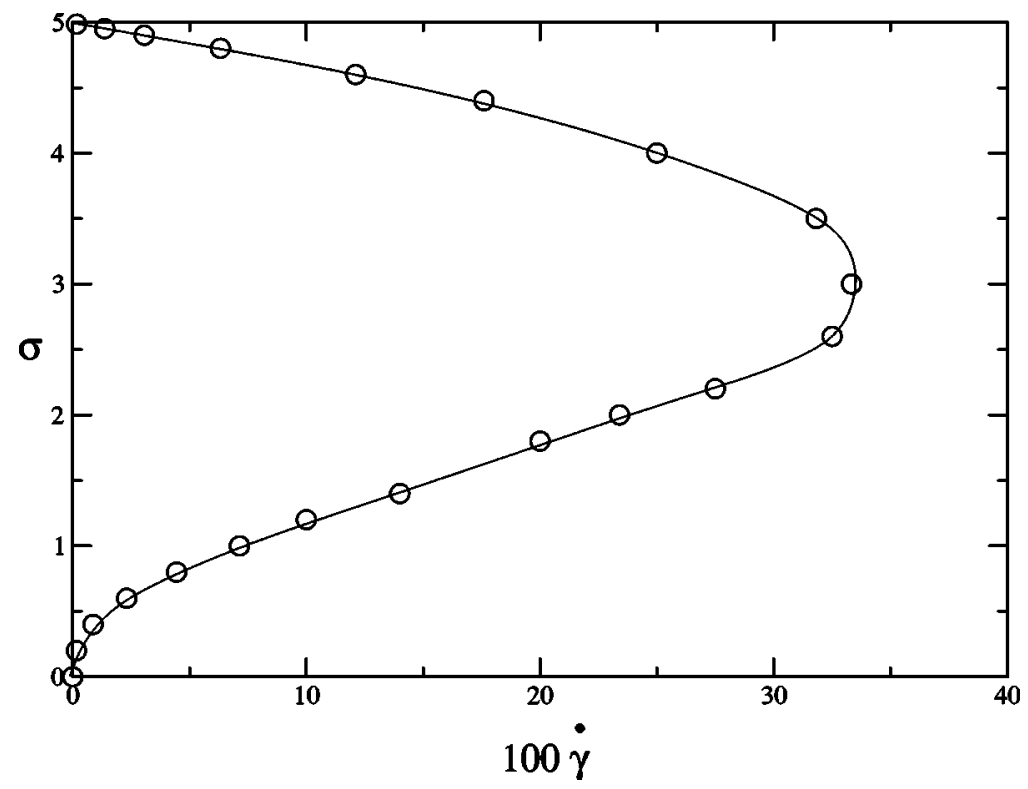

FIG. 13. Model II flow curves for $v_{2}=3.9, \alpha=0.2$. Note the presence of thinning at low stresses, followed by thickening and jamming. No fluidization after stress-induced jamming is found for these (or any) parameters with this model.

\section{Model IV}

Model IV incorporates a nonexponential form of $f(\dot{\gamma} t)$ inspired by the full MCT calculations [Fuchs and Cates (2002)]. This has not been treated analytically, and so results were obtained solely by numerical means. We find that the rheology of this model

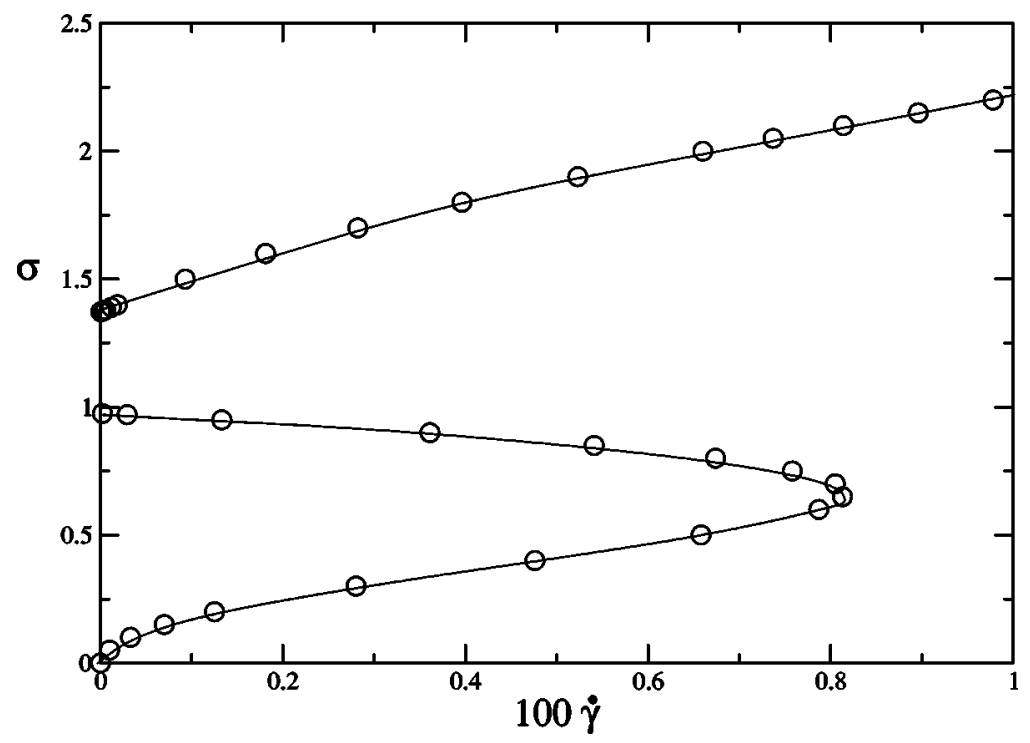

FIG. 14. The model III flow curve with $v_{2}=3.9, \alpha=0.95$. Shear thinning preceeds thickening and jamming. 


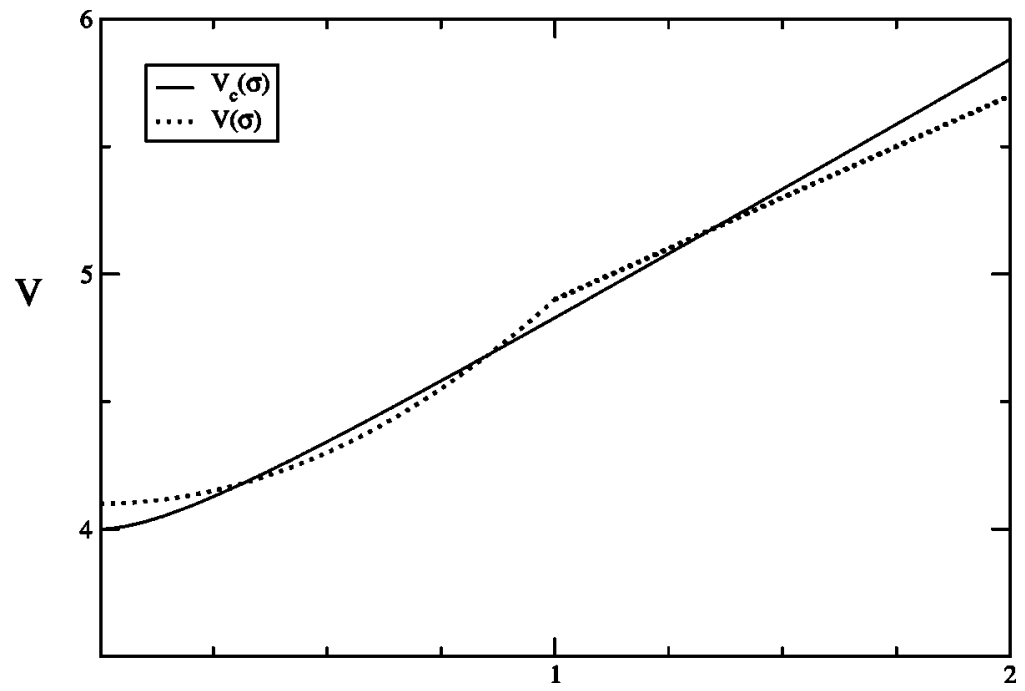

FIG. 15. The model III phase diagram in the $(\sigma, V)$ representation for $v_{2}=4.1, \alpha=0.8$. A shear melted glass (or quiescent fluid; not shown) can exhibit thickening and full jamming at larger stress, as in model II (Fig. 12). But in this case, the ultimate state at high stress is a fluid for $\alpha<1$.

is qualitatively similar to that of model I: the same shear thickening scenarios appear, and so we do not show any flow curves.

In contrast, the correlation functions do differ from those of model I. In particular, the late-time exponential decay present in models I and II does not consistently appear in this case: in Fig. 16 we show an example where the late-time correlator is not well described by an exponential. This suggests that the exponential decay of models I and II is an

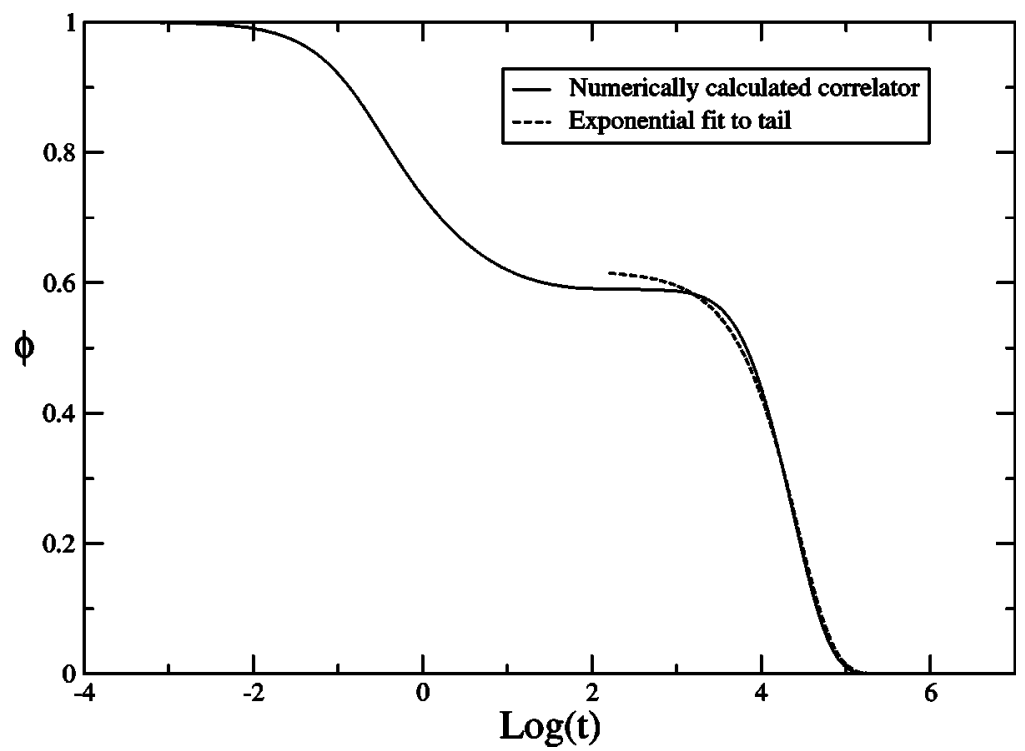

FIG. 16. The model IV correlation function for $v_{2}=3.9, \alpha=0.3$ and $\sigma=0.78$ (the system has been refluidized: $\sigma_{c 2} \approx 0.775$ ), with an exponential fit to the late-time decay. The late-time decay deviates from exponential form. 


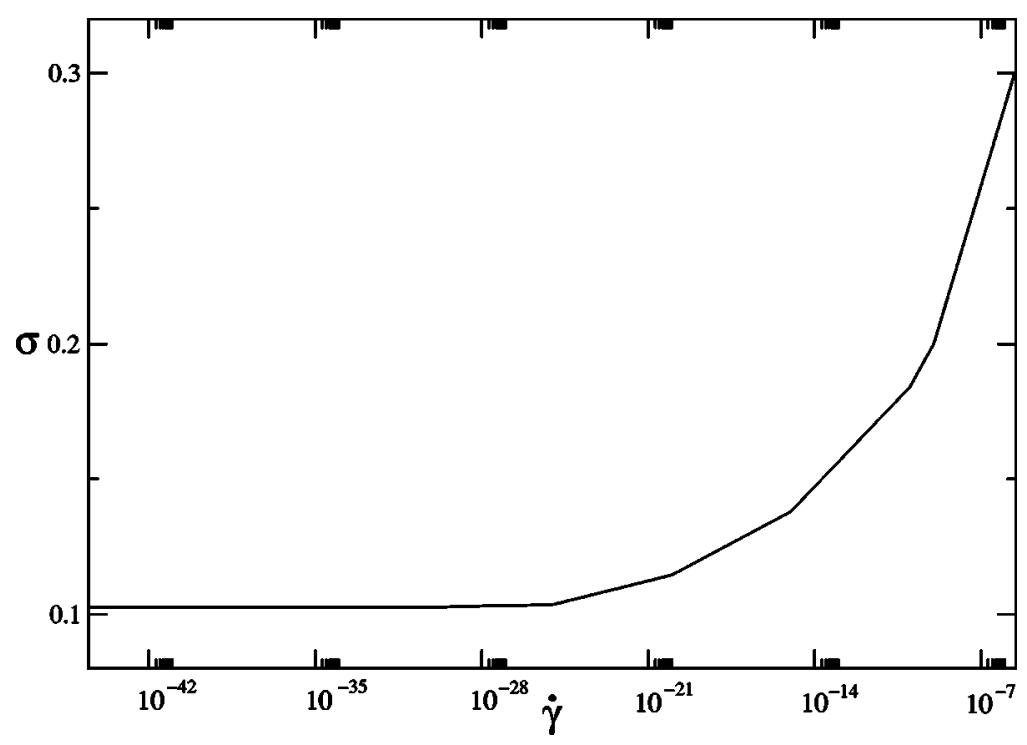

FIG. 17. The $\alpha=0$ flow curve of model IV at $v_{2}=4$. Note the logarithmic scale of the $\dot{\gamma}$ axis.

artifact of their precise form (nevertheless, it is helpful as it allows analytic progress). A second difference regards the behavior in the case $\alpha=0$. In Sec. V, we showed that, for model I, the $\alpha=0$ yield stress vanishes smoothly as $v_{2} \rightarrow 4^{+}$. Here, elucidating this point is not so simple, as we have no analytic expression for the transition points. However, numerics suggest that the yield stress does not vanish smoothly as $v_{2} \rightarrow 4^{+}$for model IV (Fig. 17). We have no explanation for the very small flow rates needed to reveal this effect; the yield stress itself is not small.

\section{Model V}

We turn finally to model $\mathrm{V}$, which differs from model I in that it is based on the F12 schematic model, rather than the simpler F2 model. This creates an extra dimension in the parameter space, since there are two coupling parameters whose variation with applied stress need not be identical-i.e., in Eq. (10), $\alpha_{1}$ need not be identical to $\alpha_{2}$. Our exploration of the parameter space has uncovered thickening and jamming scenarios qualitatively similar to that of models I and IV. Other scenarios may be present, but we have not found any which differ qualitatively from those shown earlier.

However, as in model IV, the correlation functions do not always show a late-time exponential decay in the vicinity of jamming transitions. An unresolved issue concerns nature of the $\alpha=0$ yield stress: does it appear discontinuously at the quiescent glass transition (as in model IV), or does it vanish smoothly (models I, II, and III)? We have been unable to answer this question numerically: but in contrast to model IV, the yield stress, if it exists at all, is very small $\left(\lessgtr 10^{-5}\right)$.

\section{E. Structural stability}

Having described the results of the various model variants, we now discuss the structural stability of our class of models. The shear thickening/jamming scenarios that we find appear fairly robust with respect to the precise form of the memory function, indicating that they arise generically from the competition between stress-induced jamming 
and flow-induced fluidization, rather than from the precise mathematical forms chosen here. The exception to this is the case of model II (and, more generally, any case in which the coupling parameters increase too quickly at large stress) in which fluid states are precluded at high stress regardless of proximity to the quiescent glass transition. This is unphysical: very dilute colloids do not jam under flow. However, model II is still useful in that it takes the "correct" form around $\sigma=0$, suggesting that thinning can preceed shear thickening. Model III successfully combines this with the desirable features of model I, in which fluid states at high stress are allowed, but only for $\alpha<1$. Since $\alpha$ (like $v_{2}$ ) can be concentration dependent, this allows an intriguing possibility, that shear melting of colloidal glasses (stress induced or otherwise) is precluded above a certain threshold of concentration.

In contrast, neither the exponential decay nor the smoothly appearing yield stress at $\alpha=0$ found in models I-III are robust under alterations to the chosen form of the memory function. In conventional MCT, late-time decays in the vicinity of the glass transition are not exponential, but rather are well described by the stretched exponential form $\left[e^{-(t / \tau)^{\beta}}\right.$ with the exponent $\left.\beta<1\right]$. This "dynamical stretching," which is found experimentally, is absent in the F2 model, although it reappears in F12 and other, more general, schematic models. It is not surprising, then, that model I leads to exponential decay while model IV does not. Dynamical stretching is associated with the cooperative motion required for relaxation in a dense liquid. In the vicinity of a jamming transition, relaxation occurs via smaller scale motions which are then accentuated by the flow. Thus, although our results regarding this point are inconclusive, one might expect that dynamical stretching is less pronounced near these transitions than near the quiescent glass transition [Fuchs and Cates (2003)]. If so, the assumption of an exponential decay could be a harmless one.

\section{DISCUSSION}

\section{A. The jamming transitions}

Nonergodic solutions to our schematic MCT equations are always available for $V$ $\geqslant 4$ [Eq. (15)]. However, they are not the only possible solutions. We have seen that ergodic, flowing solutions can also exist within this region: if both are present, the iteration method we used for $\tau$ [based, e.g., on Eq. (24)] leads always to the ergodic solution, and jamming transitions occur at points where these solutions cease to exist. So far, we have tacitly assumed that physical stability follows from such stability under iteration: although plausible, this is not obvious. We discuss this assumption later, but first, presuming that it holds, we compare our jamming transitions to the conventional static glass transition as described by standard MCT.

In standard MCT, glass transitions occur at points in parameter space where nonergodic solutions become available. At these so-called "critical points" [Götze (1989)], there is a bifurcation in the long-time limit $(f)$ of the correlation function: it jumps from its value in the fluid $(f=0)$ to a nonzero value $f_{c}$, followed by a nonanalytic variation as the coupling is further increased $\left(f-f_{c} \sim \epsilon^{1 / 2}\right.$, where $\epsilon$ is the separation from the transition in the space of coupling constants). At lower values of the coupling, no such nonergodic solution is possible. In the case of our jamming transitions, this is not the case: rather (at fixed $v_{2}, \alpha \ldots$ ) the system jams at values of the stress for which the ergodic, flowing solutions vanish. These points are not critical points, and do not correspond to a bifurcation in $f$. Therefore, the initial jump in $f$ at jamming transitions is followed by an analytic variation with coupling (i.e., with the stress if other parameters 


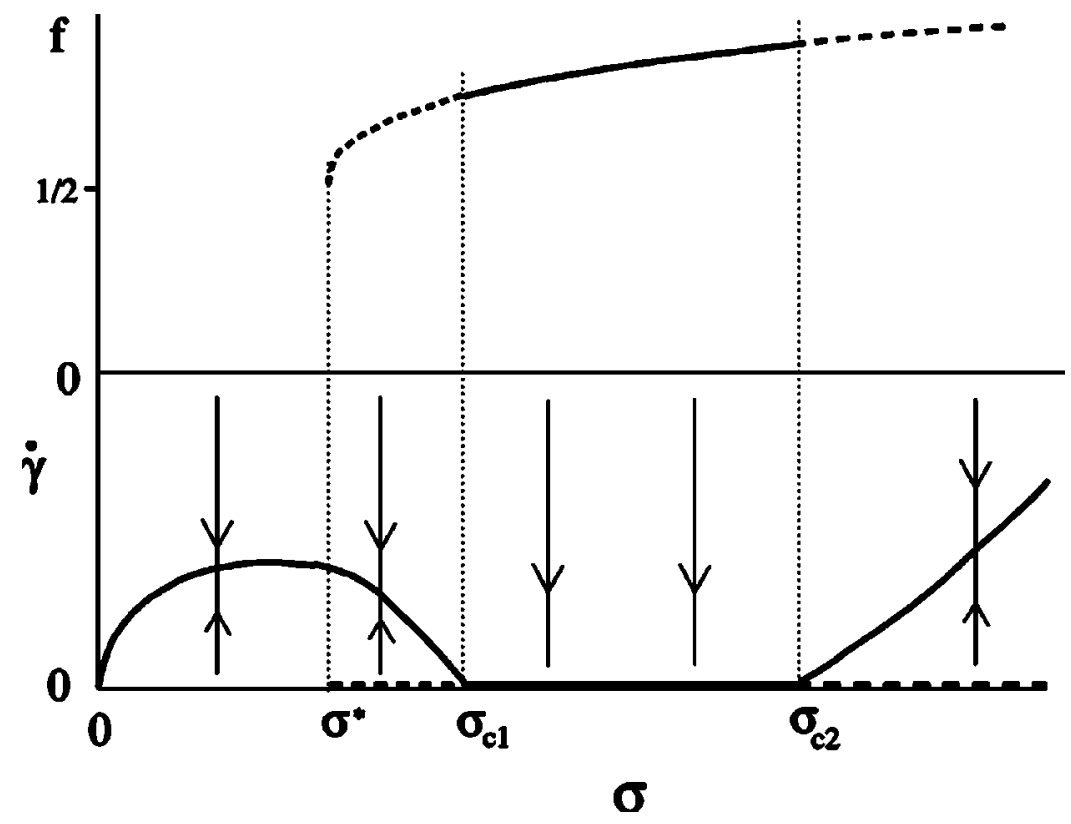

FIG. 18. Schematic variation of $\dot{\gamma}$ and $f$ with $\sigma$ (model I, $\alpha<1$, full jamming scenario). Solid and dashed lines denote stable and unstable solutions under the iteration described in Sec. IV. Arrows indicate flow of $\dot{\gamma}$ under the iteration.

are fixed). This point is illustrated by the variation of the nonergodicity parameter $f$ with $\sigma$, as shown in Fig. 18, which corresponds to one of the "full jamming" flow curves in Figs. 2 or 4 for model I.

In the figure, there is a range of stress for which an arrested solution has become available (indicated by the existence of nonzero $f$ in the upper part of the diagram) but is not chosen-rather, the system remains flowing (as indicated in the lower part by a nonzero shear rate). Only when the viscosity of the flowing system diverges (at $\sigma_{c 1}$ ), signalling that flowing solutions are no longer possible, does the system become nonergodic. At $\sigma_{c 2}$, a flowing solution again becomes available.

The fact that jamming transitions are not critical points was used in Sec. IV to guide the analysis presented there. As discussed also in Sec. V C, the $\beta$-relaxation time $\tau_{\beta}$, and not just the longer $\alpha$-relaxation, diverges at a critical point in MCT, whereas at a generic jamming transition $\tau_{\beta}$ remains finite. This means that, by moving close enough to a jamming transition, we can make the shear rate sufficiently small that $\dot{\gamma} \tau_{\beta} \ll 1$ so that the underlying $\beta$-relaxation is essentially unperturbed by the flow. This gives a preplateau relaxation of the correlator on time scale $\tau_{\beta}$, followed at time $\tau \sim 1 / \dot{\gamma}$ by the flow induced terminal relaxation. This leads to a very well defined plateau region, visible in Fig. 10, which is quite different from a quiescent MCT scenario.

This prediction is testable in principle, although the advection of density fluctuations makes the measurement of correlators somewhat tricky. One idea is to monitor the decay of density fluctuations with wavevector along the neutral (vorticity) direction. These fluctuations are not advected by the flow, so that there is some chance of observing the characteristic properties of the correlators just described, for example at stresses just above $\sigma_{c 2}$. 


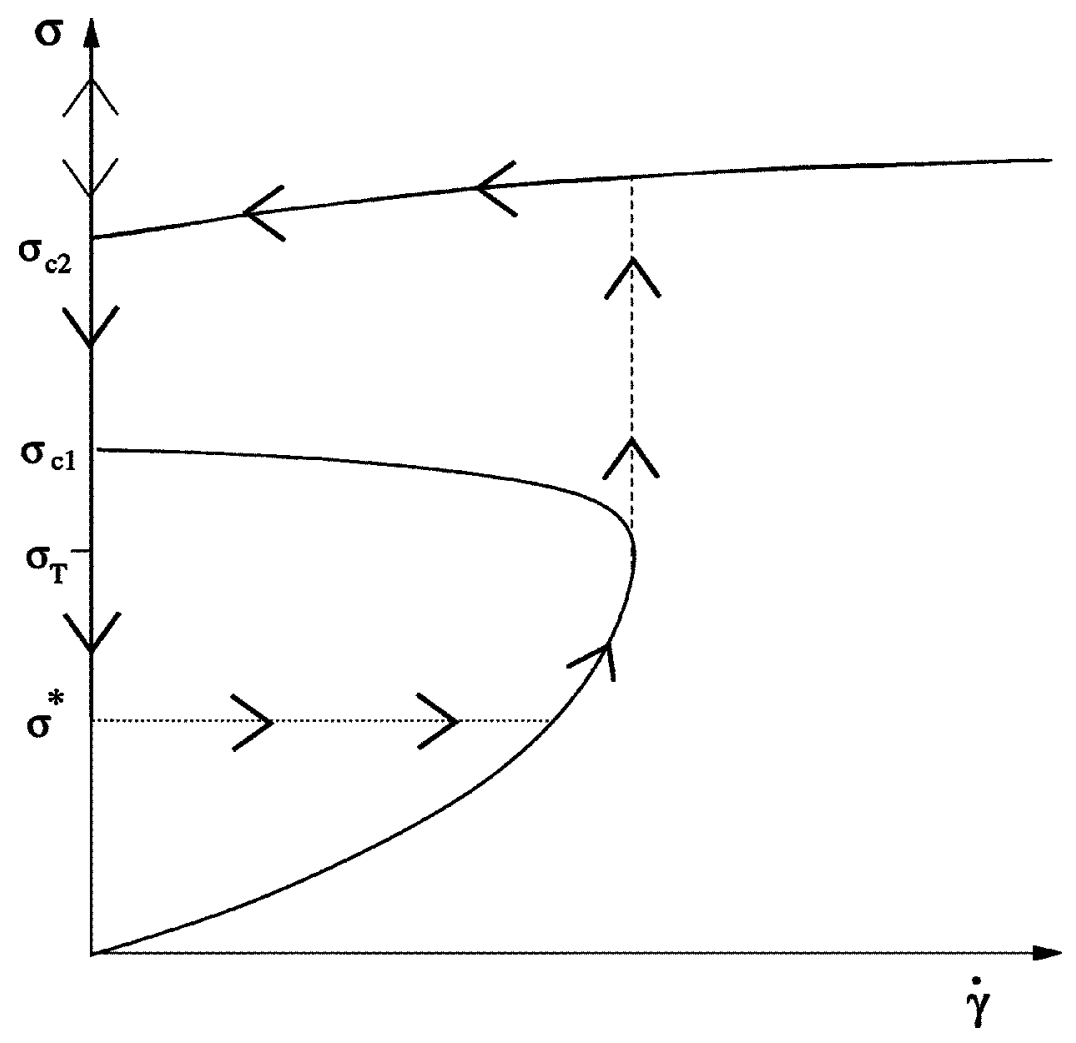

FIG. 19. Full jamming scenario of model I, showing in bold the full manifold of steady state MCT solutions, including nonergodic solutions that are iteratively unstable (these lie on the vertical axis, outside the window $\sigma_{c 1}<\sigma<\sigma_{c 2}$ ). Vertical dashed line: extreme possible discontinuous shear-thickening curve arising from shear-banding. Bold arrows: extreme possible hysteresis loop. Light arrows: possible exploration of further jammed states.

\section{B. Physical stability of solutions}

We have so far assumed that stability of solutions to our MCT equations under iteration [e.g., of Eq. (24)] is equivalent to physical stability of those solutions. There are two ways in which this assumption can fail. One is well understood, and relates to the mechanical instability of flow curves of negative slope with respect to shear banding. We discuss this later (Sec. VII B 2), but first address a different question-that of selection between different solutions of the schematic MCT equations when more than one exists.

\section{Iterative versus physical stability}

As mentioned earlier, iterative stability selects ergodic solutions over nonergodic ones, when both exist. Although such iteration does not directly map onto temporal evolution of the shear rate, one could imagine a transient violation of Eq. (6), resulting in an infinitesimal shear rate arising in an otherwise nonergodic state. Assuming a correspondence between iteration and time evolution, this would carry the system to the flowing solution whenever it exists; the arrows in Fig. 18 would be genuine trajectories. Without knowing this correspondence, however, it seems wise to allow that when nonergodic and flowing solutions of our models both exist mathematically, either or both could be locally stable. For the full jamming scenario of Fig. 18 and Figs. 2 and 4, this leads to the manifold of steady state solutions depicted by the bold line in Fig. 19. 
A pessimistic view, on the other hand, is that the nonergodic solution is the only physically stable one whenever it does exist (so that physical stability is the opposite of iterative stability). This assumption would destroy much of the interesting rheology we have reported; for example, the full jamming scenario seen in Fig. 19 would be replaced by a flow curve that consists of the lowest branch of the S-shaped flow curve for stresses less than $\sigma^{*}$, then jumps discontinuously back to $\dot{\gamma}=0$ (dotted line) and remains there for all $\sigma>\sigma^{*}$ (vertical bold line). This scenario cannot really explain either continuous or discontinuous shear thickening. Moreover, its presumption that a nonergodic state is always preferred to flowing alternative contradicts the usual notion that glasses are arrested because they are trapped.

\section{Mechanical instability and hysteresis}

Let us assume instead that the full manifold of steady-state solutions are candidates for physical stability. These solutions describe putatitive steady, homogeneous states; however, it is well known that any flow curve containing regions of negative slope is unstable to shear banding [Dhont (1999), Olmsted (1999)]. For shear thickening systems the bands comprise slabs of material oriented layerwise in planes perpendicular to the vorticity (neutral) axis; these slabs have a common shear rate but different stresses. \{The shear thinning case, in which layers are oriented parallel to the sliding walls of a couette, may be more familiar [Olmsted (1999), Spenley et al. (1993)].\} In a rheometrical experiment with solid walls, these stresses get averaged over the slabs; thus, even a stress-controlled experiment controls only this average.

Accordingly the part of the solution manifold in Fig. 19 between $\sigma_{c 1}$ on the vertical axis and the point of vertical tangency to the flow curve (at stress $\sigma_{T}$ ) is mechanically unstable and will not be observed in steady state, even in a controlled-stress rheometer. The standard shear banding scenario then predicts that if a quiescent fluid is progressively sheared with increasing $\dot{\gamma}$, at some point before the vertical tangent is reached the stress will jump onto the upper branch of the flow curve. This is an extreme limit of stability; in practice it could jump sooner, at a shear rate that could depend on various aspects of sample history. [Such phenomena are well studied in the shear-thinning case; see Grand et al. (1997).] Thus, shear banding explains discontinuous shear thickening as the natural consequence of an S-shaped flow curve. If $\dot{\gamma}$ is increased further, the upper branch will be followed out to the right.

If $\dot{\gamma}$ is now slowly reduced, there can be hysteresis, in which the jump back to the lower branch is delayed. For an S-shaped curve that does not cross the axis (see Fig. 2 for examples), this must occur at or before a second point of vertical tangency, and the hysteresis loop is of limited width \{as reported experimentally [Frith et al. (1996), Laun (1994)]\}. However, in the full jamming curve of Fig. 19 it becomes possible in principle to track right back to the vertical axis, achieving the jammed state of zero shear rate but a finite stress. This offers one way to access the jamming transition at $\sigma_{c 2}$ and the stress-induced glass at $\sigma \leqslant \sigma_{c 2}$. Another is to perform a stress-controlled measurement in the window between $\sigma_{c 1}$ and $\sigma_{c 2}$; however, since the controlled stress is a spatial average, this might lead to a banded flow instead (corresponding, e.g., to a point on the vertical dashed line in Fig. 19). Once the vertical axis has been reached, it should be possible to vary the static stress, at least within the window of full jamming. It should also be possible to see whether the nonergodic states outside this window are locally stable; if they are, the stress could be increased indefinitely (in principle!) without steady flow arising, and/or reduced as far as $\sigma^{*}$. The latter sets the lower left corner of an extreme possible hysteresis loop, as indicated in Fig. 19. Note that we have entirely 
excluded sublinear creep from these discussions which address only steady-state flow and its absence; in practice creep could complicate matters considerably.

Note also that if a model is chosen with no upper branch to the flow curve, as in Fig. 5 (model I with $\alpha>1$ ) the preceding discussion of hysteresis might still apply so long as such a branch is furnished by some other mechanism, such as fracture. Experimentally, it often reported than flow in the strong shear thickening regime is far from steady [Frith et al. (1996)]; this might indeed be consistent with a flow curve such as Fig. 5, plus an unsteady fracture mechanism at high stresses. This hybrid mechanism would allow a complete separation of stress scales between the upper (shear-thickened) part of the effective flow curve and the lower (quasi-Newtonian) part; in particular, the upper stress level could vastly exceed the intrinsic modulus scale for a colloidal glass. The latter is of order $k_{B} T / a^{3}$ close to the arrest transition, which is of order 1-100 $\mathrm{Pa}$ for typical parameters and decreasing with particle size. Much higher stresses are indeed sometimes reported in jammed colloids [d'Haene et al. (1993), Frith et al. (1996)].

\section{Comparison with experimental jamming transitions}

As discussed in Sec. I, phenomena reminiscent of our jamming transitions have been observed experimentally. In the work of Bertrand et al. (2002), jamming occurred only for a particular range of concentrations: at lower volume fractions, shear thickening was observed, while at higher concentrations, the sample was solid in its quiescent state. A similar sequence arises in our model $\mathrm{I}$ if $v_{2}$ increases with concentration (as it surely does) and $\alpha$ remains less than unity. Thus it is tempting to identify the jamming transitions of our model with the stress-induced solids recently found experimentally [Bertrand et al. (2002), Haw (2004)].

However, such an identification is uncertain. The particles used in the experiments of Bertrand et al. (2002) are perhaps too large for glass transition concepts to be relevant; they have radius $a \sim 3 \mu \mathrm{m}$, so that Brownian forces are small compared with forces such as gravity (no density matching was done) and a purely mechanical description might be more appropriate. The particles which most easily jam in the experiments of Haw (2004) are also at the upper end of the colloidal size range, with radii $a \sim 1 \mu \mathrm{m}$; it would be of great interest to study shear thickening with particle sizes smaller than this. Also, most experiments on dense suspension rheology do not utilize the clean model systems latterly favored by colloid physicists [Haw (2004), Pham et al. (2002), Watanabe et al. (1999)]. It is possible to closely approximate hard-sphere interactions (this generally requires optical index matching with the solvent) to which attractive forces can then be added controllably. Further investigations of shear thickening in such model systems would be particularly welcome; ideally one would like to know the behavior as a function of concentration, particle size, and interactions. One could then aim to establish a rational correlation between these and parameters such as $v_{2}$ and $\alpha$ in our models.

\section{CONCLUSIONS}

The rheology of concentrated suspensions is sufficiently complex that a full microscopic theory is not yet feasible. Deliberately simplified models can prove useful in improving our understanding of these materials. In this article we have presented a family of models of the rheological behavior of very dense suspensions, motivated by the modecoupling theory of the glass transition, but neglecting hydrodynamics.

Upon variation of model parameters, a wide range of rheological behavior was found, ranging from shear thinning, through continuous and discontinuous thickening (the latter interpreted as shear banding), to a jamming transition into a nonergodic solid state. 
Depending on model parameters, this may or may not be refluidized again at higher stresses; in cases where the model predicts no refluidization one might expect another mechanism, such as fracture and/or unsteady flow, to intervene. (There is some experimental evidence for this at very high concentrations.) Various hysteresis scenarios involving jammed states and/or shear banding are also possible.

The main parameters in our simplest model variant (model I) are $v_{2}$, which is a measure of the distance from the glass transition in the quiescent state (this lies at $v_{2}$ $=4$ ), and a coupling constant $\alpha$ which controls, in effect, the stress dependence of this distance. There is also some freedom in how we model the role of strain in eroding memory, but these details are less important to the results.

The jamming transitions within our models differ from static glass transitions, in that the transition points are not critical points of our schematic MCT: the $\beta$-relaxation time does not diverge at a jamming transition. The resulting variation of the nonergodicity parameter $f$ at jamming has a discontinuity but is analytic beyond that, unlike the MCT glass transition which has a square-root contribution. Finally, in models I-III, the late time decay of correlators close to a jamming transition is exponential. However, as discussed in Sec. VIE, this is not robust but depends on the particular form of these models. Regardless of this, in all the scenarios suggested in this work, we expect that this final relaxation should differ from that close to a conventional glass transition, since the two relaxations occur in quite different ways.

Although the models presented for shear thickening and jamming remain both schematic and provisional, they may help guide future progress with more formal MCT-based developments [Fuchs and Cates $(2002,2003)]$. The role of hydrodynamic interactions in shear thickening, neglected by us, could perhaps be elucidated further by comparing Stokesian [Brady (1996)] and Brownian [Strating (1999)] dynamics simulations at very high colloidal volume fractions. It is possible that in future a version of MCT can be created that does not ignore many-body hydrodynamics, but a clearer empirical understanding of the interplay of these with the glass transition is probably needed first.

We hope that our work will promote a more careful experimental examination of the flow behavior of very concentrated suspensions, preferably in systems of small, densitymatched particles (so that Brownian motion is strong and gravity weak) with wellcontrolled colloidal interactions. The possible interplay between the jamming we describe and capillary forces at the surface of a droplet, or at the fracture surface of a bulk sample ruptured by shear, remains an open topic for future study. Finally, in the longer term, it would be very useful to develop a MCT-like theory, even at the schematic level, that can address time-dependent phenomena as well as the steady-state properties addressed in this work.

\section{ACKNOWLEDGMENTS}

The authors thank Patrick Warren, Mark Haw, Jérôme Bibette, Norman Wagner, and Thomas Voigtmann for useful discussions. This work was supported by the Engineering and Physical Science Research Council (EPSRC), through Grant No. GR/S10377/01, and by the Deutsche Forschungsgemeinschaft through Grant No. Fu309/3.

\section{References}

Ball, R. C., and J. R. Melrose, "Lubrication breakdown in hydrodynamic simulations of concentrated colloids," Adv. Colloid Interface Sci. 59, 19-30 (1995).

Barrat, J.-L., and L. Berthier, "Fluctuation-dissipation relation in a sheared fluid," Phys. Rev. E 63, 012503 (2000). 
Bender, J., and N. J. Wagner, "Reversible shear thickening in monodisperse and bidisperse colloidal dispersions," J. Rheol. 40, 899-916 (1996).

Bergenholtz, J., J. F. Brady, and M. Vicic, “The non-Newtonian rheology of dilute colloidal suspensions," J. Fluid Mech. 456, 239-275 (2002).

Berthier, L., J.-L. Barrat, and J. Kurchan, “A two-time-scale, two-temperature scenario for nonlinear rheology,” Phys. Rev. E 61, 5464-5472 (2000).

Bertrand, E., J. Bibette, and V. Schmitt, "From shear thickening to shear-induced jamming," Phys. Rev. E 66, 60401(R) (2002).

Bouchaud, J.-P., L. Cugliandolo, J. Kurchan, and M. Mezard, "Mode-coupling approximations, glass theory and disordered systems," Physica A 226, 243-273 (1996).

Brady, J. F., "Model hard-sphere dispersions: statistical mechanical theory, simulations and experiments," Curr. Opin. Colloid Interface Sci. 1, 472-480 (1996).

Cates, M. E., “Arrest and flow of colloidal glasses," Ann. Henri Poincare 4, S647-S661 (2003).

Cates, M. E., and P. Sollich, "Tensorial constitutive models for disordered foams, dense emulsions and other soft nonergodic materials," J. Rheol. 48, 193-207 (2004).

Cates, M. E., J. P. Wittmer, J.-P. Bouchaud, and P. Claudin, "Jamming, force chains and fragile matter," Phys. Rev. Lett. 81, 1841-1844 (1998).

d'Haene, P., J. Mewis, and G. G. Fuller, "Scattering dichroism measurements of flow-induced structure of a shear thickening suspension," J. Colloid Interface Sci. 156, 350-358 (1993).

Dhont, J. K. G., “A constitutive relation describing the shear-banding transition,” Phys. Rev. E 60, 4534-4544 (1999).

Fielding, S. M., P. Sollich, and M. E. Cates, “Aging and rheology in soft materials," J. Rheol. 44, 323-369 (2000).

Foss, D. R., and J. F. Brady, "Structure, diffusion and rheology of brownian suspensions by stokesian dynamics simulation," J. Fluid Mech. 407, 167-200 (2000).

Frith, W. J., P. d'Haene, R. Buscall, and J. Mewis, "Shear thickening in model suspensions of sterically stabilized particles," J. Rheol. 40, 531-548 (1996).

Fuchs, M., and M. E. Cates, "Theory of nonlinear rheology and yielding of dense colloidal suspensions," Phys. Rev. Lett. 89, 248304 (2002).

Fuchs, M., and M. E. Cates, "Schematic models of dynamic yielding of sheared colloidal suspensions," Faraday Discuss. 123, 267-286 (2003).

Fuchs, M., W. Götze, I. Hofacker, and A. Latz, "Comments on the $\alpha$-peak shapes for relaxation in supercooled liquids,” J. Phys.: Condens. Matter 3, 5047-5071 (1991).

Götze, W., "Aspects of structural glass transitions," in Liquids, Freezing and the Glass Transition, edited by J. P. Hansen, D. Levesque, and J. Zinn-Justin (North-Holland, Amsterdam, 1989), pp. 287-503.

Götze, W., "The essentials for the mode-coupling theory for glassy dynamics," Condens. Matter Phys. 1, 873-904 (1998).

Götze, W., and L. Sjögren, "General properties of certain nonlinear integrodifferential equations," J. Math. Anal. Appl. 195, 230-250 (1995).

Grand, C., J. Arrault, and M. E. Cates, "Slow transients and metastability in wormlike micelle rheology," J. Phys. II 8, 1071-1086 (1997).

Hansen, J. P., and I. R. McDonald, The Theory of Simple Liquids, 1st ed. (Academic, London, 1976).

Haw, M. D., “Jamming, two-fluid behaviour and 'self-filtration' in concentrated particulate suspensions," Phys. Rev. Lett. 92, 185506 (2004).

Head, D. A., A. Ajdari, and M. E. Cates, "Jamming, hysteresis and oscillation in scalar models for shear thickening," Phys. Rev. E 64, 061509 (2001).

Head, D. A., A. Ajdari, and M. E. Cates, "Rheological instability in a simple shear-thickening model," Europhys. Lett. 57, 120-126 (2002).

Hess, O., and S. Hess, "Nonlinear fluid behaviour-From shear thinning to shear thickening," Physica A 207, 517-540 (1994).

Hinch, E. J., Perturbation Methods (Cambridge University Press, Cambridge, 1991).

Hoffman, R. L., "Explanations for the cause of shear thickening in concentrated colloidal suspensions," J. Rheol. 42, 111-123 (1998).

Holmes, C. B., M. Fuchs, and M. E. Cates, "Jamming transitions in a schematic model of transition rheology," Europhys. Lett. 63, 240-246 (2003).

Laun, H. M., "Normal stresses in extremely shear thickening polymer dispersions," J. Non-Newtonian Fluid Mech. 54, 87-108 (1994).

Liu, A. J., and S. R. Nagel, "Nonlinear dynamics_Jamming is not just cool anymore," Nature (London) 396, 21-22 (1998).

Meeker, S. P., W. C. K. Poon, and P. N. Pusey, "Concentration dependence of the low-shear viscosity of suspensions of hard-sphere colloids," Phys. Rev. E 55, 5718-5722 (1997).

Melrose, J. R., "Colloid flow during thickening-A particle level understanding for core-shell particles," Faraday Discuss. 123, 355-368 (2003). 
Melrose, J. R., and R. C. Ball, "The pathological behavior of sheared hard-spheres with hydrodynamic interactions," Europhys. Lett. 32, 535-540 (1995).

Melrose, J. R., J. H. van Vliet, and R. C. Ball, "Continuous shear thickening and colloid surfaces," Phys. Rev. Lett. 77, 4660-4663 (1996).

Miyazaki, K., and D. R. Reichmann, "Molecular hydrodynamic theory of supercooled liquids and colloidal suspensions under shear," Phys. Rev. E 66, 050501(R) (2002).

O'Brien, V. T., and M. E. Mackay, "Stress components and shear thickening of concentrated hard sphere suspensions," Langmuir 16, 7931-7938 (2000).

Olmsted, P. D., "Dynamics and flow-induced phase separation in polymeric fluids," Curr. Opin. Colloid Interface Sci. 4, 95-100 (1999).

Pham, K. N., J. Bergenholtz, S. U. Egelhaaf, A. Moussaid, P. N. Pusey, A. B. Schofield, M. E. Cates, M. Fuchs, and W. C. K. Poon, "Multiple glassy states in a simple model system," Science 296, 104-106 (2002).

Phung, T. N., J. F. Brady, and G. Bossis, "Stokesian dynamics simulation of brownian suspensions," J. Fluid Mech. 313, 181-207 (1996).

Pusey, P. N., "Colloidal suspensions," in Liquids, Freezing and the Glass Transition, edited by J. P. Hansen, D. Levesque, and J. Zinn-Justin (North-Holland, Amsterdam, 1989), pp. 763-942.

Sollich, P., F. Lequeux, P. Hebraud, and M. E. Cates, "Rheology of soft glassy materials," Phys. Rev. Lett. 78 2020-2023 (1997).

Spenley, N. A., M. E. Cates, and T. C. B. McLeish, "Nonlinear rheology of wormlike micelles," Phys. Rev. Lett. 71, 939-942 (1993).

Strating, P., "Brownian dynamics simulation of a hard sphere suspension," Phys. Rev. E 59, 2175-2187 (1999).

van Megen, W., and S. M. Underwood, "Glass transition in colloidal hard spheres-Measurement and modecoupling theory analysis of the coherent intermediate scattering function," Phys. Rev. E 49, 4206-4220 (1994).

Warren, P. B. (private communication).

Watanabe, H., M. L. Yao, K. Osaki, T. Shikata, H. Niwa, and Y. Morishima, "Nonlinear rheology of concentrated spherical silica suspensions: 3. Concentration dependence," Rheol. Acta 38, 2-13 (1999). 